\title{
Edge-magnetoplasmon wave-packet revivals in the quantum-Hall effect
}

\author{
U. Zülicke \\ Department of Physics, Indiana University, Bloomington, Indiana 47405 \\ Robert Bluhm \\ Department of Physics, Colby College, Waterville, Maine 04901 \\ V. Alan Kostelecký and A. H. MacDonald \\ Department of Physics, Indiana University, Bloomington, Indiana 47405
}

(Received 5 December 1996)

\begin{abstract}
The quantum-Hall effect is necessarily accompanied by low-energy excitations localized at the edge of a two-dimensional electron system. For the case of electrons interacting via the long-range Coulomb interaction, these excitations are edge magnetoplasmons. We address the time evolution of localized edge-magnetoplasmon wave packets. On short times the wave packets move along the edge with classical $E$ cross $B$ drift. We show that on longer times the wave packets can have properties similar to those of the Rydberg wave packets that are produced in atoms using short-pulsed lasers. In particular, we show that edge-magnetoplasmon wave packets can exhibit periodic revivals in which a dispersed wave packet reassembles into a localized one. We propose the study of edge-magnetoplasmon wave packets as a tool to investigate dynamical properties of integer and fractional quantum-Hall edges. Various scenarios are discussed for preparing the initial wave packet and for detecting it at a later time. We comment on the importance of magnetoplasmon-phonon coupling and on quantum and thermal fluctuations. [S0163-1829(97)07716-3]
\end{abstract}

\section{INTRODUCTION}

The quantum-Hall (QH) effect occurs in two-dimensional (2D) electron systems (ES's) whenever the chemical potential has a discontinuity which occurs at a magnetic-fielddependent density. ${ }^{1}$ For isotropic 2 D ES's, charge gaps occur at integer and certain noninteger but rational values of the filling factor $\nu:=(n / B) \Phi_{0}$, where $\Phi_{0}=h c / e$ is the magnetic-flux quantum. The origin of the charge gap in the case of the integral filling factor $\nu$ is the Landau-level quantization of single-electron orbits in a magnetic field, and the gap is therefore of the order of the cyclotron energy. For the most part, we will assume in this paper that the 2D ES has a filling factor that is the inverse of an odd integer: $\nu=1 / \mathrm{m}$, with $m$ odd. If $m>1$, the charge gap arises from the Coulomb interaction between the electrons in the QH sample and is typically smaller than the cyclotron energy. When the $\mathrm{QH}$ effect occurs, the bulk of the system is incompressible in the absence of disorder, and the only gapless excitations are localized near the boundary of the finite $\mathrm{QH}$ sample. ${ }^{2}$ For $\nu=1 / \mathrm{m}$ and the case of a sharp edge, i.e., a confining potential that varies rapidly on a length scale of the order of the magnetic length $\ell=\sqrt{\hbar c /|e B|}$, the edge excitations are expected $^{3}$ to be well described by a chiral Luttinger liquid (CLL) theory ${ }^{4-6}$ with a single branch of chiral bosons. In this theory, the edge of a two-dimensional electron system is thought of as a one-dimensional electron gas, ${ }^{7-9}$ which can be studied using bosonization techniques. The only lowlying excitations are collective bosonic density waves. The CLL picture can be applied if the energy of these excitations is much smaller than the charge gap. The wavelength of the lowest-lying bosonic density waves is of the order of the system size. For sharp $\nu=1 / m$ edges in the quantum-Hall regime, it turns out ${ }^{2,4}$ that there is a single branch of bosons and that these are chiral, i.e., they occur with only one sign of the wave vector. If a long-range Coulomb interaction is present, the bosons are called edge magnetoplasmons ${ }^{10-12}$ (EMP's). It is possible to derive an expression for the energy dispersion relation of edge magnetoplasmons starting from the microscopic Hamiltonian for electrons moving in a strong magnetic field and interacting via a 3D Coulomb interaction. ${ }^{13}$ The result, which is valid for both the disk and strip geometries, is

$$
\varepsilon_{M}^{C}=-\frac{M}{R} \ln \left[\alpha \frac{M}{R}\right] .
$$

Expressions differing from Eq. (1) only in the constant $\alpha$ had been obtained earlier using a semiclassical approach. ${ }^{12,14,15}$ Here, the constant $\alpha$ is of order unity and depends weakly on the geometry of the $\mathrm{QH}$ sample, $M$ is a positive integer, ${ }^{16}$ and $R$ is related to the perimeter $L$ of the $\mathrm{QH}$ sample: $R=L / 2 \pi$. For the disk geometry, $R$ is the radius of the disk. In Eq. (1), as well as in all expressions to follow in this paper, we measure physical quantities in quantum-Hall units to simplify expressions. These units are defined in Table I.

We can think of the edge of a $\mathrm{QH}$ sample as an excitable one-dimensional medium, much like a string. The edge magnetoplasmons are the eigenmodes of this medium. We do not consider coupling to bulk excitations; this approximation is well justified at low temperatures in the middle of a $\mathrm{QH}$ plateau. For the case of a short-range interaction between the electrons, the EMP dispersion is linear in the wave number: $\varepsilon_{M}^{\mathrm{sr}}=v_{F} M / R$. If a long-range Coulomb interaction is present, the dispersion relation is nonlinear and is given by Eq. (1). Using a time-dependent external potential, it is pos- 
TABLE I. Quantum-Hall units. Throughout this paper, physical quantities are measured in these units. Besides the formal expression for each quantum-Hall unit, explicit values are given (as a function of magnetic field $B$ and filling factor $\nu$ ) which apply to 2D ES's in $\mathrm{Al}_{x} \mathrm{Ga}_{1-x} \mathrm{As} / \mathrm{GaAs}$-heterostructures. The symbols $e, \hbar$, and $k_{B}$ denote the electron charge, Planck constant, and Boltzmann constant, respectively.

\begin{tabular}{lcc}
\hline \hline Quantity & QH unit (in cgs) & Values (for GaAs) \\
\hline Length & $\ell=\sqrt{\hbar c /|e B|}$ & $25.7 \times B[\mathrm{~T}]^{-1 / 2} \mathrm{~nm}$ \\
Energy & $\left(\nu e^{2}\right) /(\pi \epsilon \ell)$ & $1.39 \times \nu B[\mathrm{~T}]^{1 / 2} \mathrm{meV}$ \\
Time & $(\hbar \pi \epsilon \ell) /\left(\nu e^{2}\right)$ & $4.74 \times 10^{-13} / \nu B[\mathrm{~T}]^{1 / 2} \mathrm{~s}$ \\
Temperature & $\left(\nu e^{2}\right) /\left(\pi \epsilon \ell k_{B}\right)$ & $16 \times \nu B[\mathrm{~T}]^{1 / 2} \mathrm{~K}$ \\
\hline \hline
\end{tabular}

sible to excite a superposition of these eigenmodes. In this way, a wave packet can be created for the electron number density along the edge. We refer to these as edgemagnetoplasmon wave packets (EMP WP's).

In this paper, we examine the formation and evolution of EMP WP's in two-dimensional electron systems. Experimental studies ${ }^{17-24,10,14}$ of EMP's have employed different excitation processes, including capacitive coupling ${ }^{21}$ or Ohmic contacts ${ }^{22}$ between the $\mathrm{QH}$ edge and an exciting voltage pulse. This paper is motivated most strongly by the capacitive coupling ${ }^{21}$ approach and we comment later on the interpretation of these experiments. The physical observables involved in these probes can all be expressed in terms of the operators involving the creation and annihilation operators for bosonic density fluctuations (EMP's): $b_{M}^{\dagger}$ and $b_{M}$. In particular, the one-dimensional number density of electrons at the edge of a sample with filling factor $\nu$, defined by integrating perpendicular to the edge and comparing with the ground-state density, is related to boson creation and annihilation operators by ${ }^{6}$

$$
\varrho(\theta)=\sum_{M>0} \frac{(\nu M)^{1 / 2}}{2 \pi R}\left[b_{M} e^{i M \theta}+b_{M}^{\dagger} e^{-i M \theta}\right]
$$

In this expression, we have fixed the direction of the magnetic field. Reversing the field direction corresponds to interchanging $b_{M}^{\dagger}$ and $b_{M}$. In our convention, the EMP's travel counterclockwise. The experimental configuration is shown in Fig. 1.

Localized electron wave packets have been produced and studied in atoms using short-pulsed lasers. ${ }^{25-29}$ A superposition of highly excited or Rydberg states is formed when a short laser pulse coherently excites a single electron far from the ground state of an atom. The resulting Rydberg wave packet is localized spatially, and its initial motion mimics the classical periodic motion of a charged particle in a Coulomb potential. The wave packet will eventually disperse and lose its classical character. However, the wave packet reassembles at later times in a sequence of fractional and full revivals and super revivals. ${ }^{25,26,30-32}$ The revivals result from quantum interference between the different eigenstates in the superposition. The appearance of revivals is quite general and can occur in quantum systems other than Rydberg atoms, ${ }^{33}$ including systems with eigenenergies depending on more than one quantum number. ${ }^{34}$

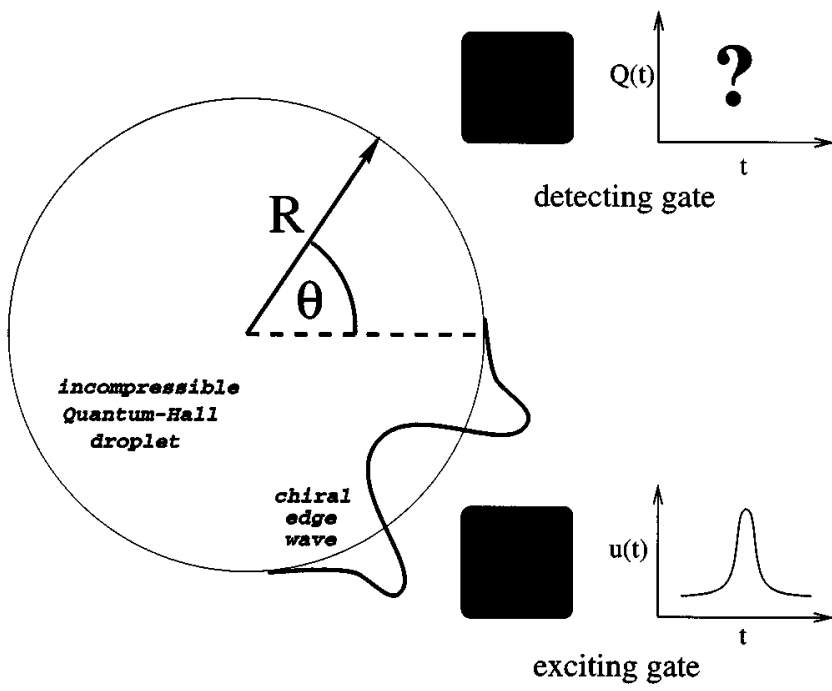

FIG. 1. Schematic picture of a chiral-edge-wave excitation and detection. A voltage pulse is applied to the exciting gate, leading to the formation of an edge-magnetoplasmon wave packet, i.e., a superposition of edge-magnetoplasmon (EMP) modes. This wave packet propagates according to the dynamics of EMP and can be detected at a second gate. The study of the detected pulse provides the opportunity to investigate the dynamical properties of EMP modes as well as the revival structure of edge-magnetoplasmon wave packets.

In the present work, we perform an analysis of the revival structure of EMP WP's in two-dimensional electron systems that on short times exhibit classical $E$ cross $B$ drift motion in the electric field that confines the electronic system to a finite area. At longer times these wave packets disperse but we show that they can also have fractional and full revivals and super revivals.

We begin in Sec. II with a brief review of Rydberg wavepacket behavior, including a discussion of fractional and full revival of dispersed wave packets. Section III describes the dynamics of EMP's in QH samples with sharp edges. The exact solution of the time-evolved edge-magnetoplasmon wave packet is obtained. We demonstrate that EMP WP's can exhibit full and fractional revivals that we illustrate for one particular set of experimental parameters. The similarities between Rydberg wave packets and EMP WP's are examined. In Sec. IV, we discuss experimental issues, taking into account the decay of EMP WP's due to scattering processes occurring in the semiconductor host material. Our conclusions are presented in Sec. V. Some details of the derivations are given separately in the appendixes.

\section{ATOMIC RYDBERG WAVE PACKETS}

In this section, we present some background on atomic Rydberg wave packets. These wave packets form when a short-pulsed laser excites an atomic electron into a coherent superposition of large-quantum-number Rydberg states. Several theoretical approaches have been used to investigate Rydberg wave packets. They have been studied both numerically ${ }^{25}$ and perturbatively. ${ }^{26}$ A description in terms of squeezed states has also been given. ${ }^{35}$ Rydberg wave packets offer the possibility of approaching the classical limit of mo- 
tion for electrons in atoms. Initially, the motion of a Rydberg wave packet is semiclassical, exhibiting the classical periodic motion of a charged particle in a Coulomb field. The period of the motion is the classical keplerian period $T_{\mathrm{cl}}$. This semiclassical motion typically lasts for only a few cycles, after which the wave packet disperses and collapses. However, quantum interference effects subsequently cause the wave packet to undergo a sequence of fractional and full revivals.

The full revivals are characterized by the recombination of the collapsed wave packet into a form that resembles the initial wave packet. This first occurs at a time $t_{\text {rev }}$. At the full revival, the wave packet again oscillates with the classical period $T_{\mathrm{cl}}$. The fractional revivals occur at earlier times equal to irreducible rational fractions of $t_{\text {rev }}$. At the fractional revivals, the wave packet separates into a set of equally weighted subsidiary wave packets. The motion of the subsidiary wave packets is periodic with the period equal to a rational fraction of the classical period $T_{\mathrm{cl}} \cdot{ }^{30}$ Eventually the periodic revivals fail, but on a still longer time scale they can reappear and a higher level of revival structure commences. ${ }^{32}$ This occurs on a longer time scale called the super revival time $t_{\mathrm{sr}}$. At times equal to certain rational fractions of $t_{\mathrm{sr}}$, distinct subsidiary waves form again, but with a period equal to a rational fraction of $t_{\text {rev }}$. These long-term fractional revivals culminate with the formation of a super revival at the time $t_{\mathrm{sr}}$. At the super revival, the wave packet can resemble the initial wave packet more closely than at the full revival time $t_{\text {rev }}$. An analysis including the super revival time scale has been performed ${ }^{32}$ both for hydrogenic models and using supersymmetry-based quantum-defect theory, ${ }^{36}$ to model the Rydberg alkali-metal atoms typically used in experiments. For times typically a few orders of magnitude greater than $t_{\mathrm{sr}}$, atomic Rydberg wave packets spontaneously decay into lower-energy states by emitting photons.

Experiments ${ }^{27-29,37}$ have studied the revival structure of Rydberg wave packets through times $\sim t_{\text {rev }}$. These experiments use a pump-probe method of detection involving either photoionization ${ }^{26}$ or phase-sensitive Ramsey interference and electric-field ionization. ${ }^{38-40}$ In both of these procedures, the wave packet is excited initially by a short laser pulse that creates a superposition of energy eigenstates with the principal quantum number centered on a value $\bar{n}$. The wave packet initially forms near the nuclear core of the atom. After the pump pulse has passed, the wave packet evolves under the influence of the Coulomb potential, oscillating between inner and outer apsidal points of the keplerian ellipse corresponding to $\bar{n}$.

In pump-probe experiments using photoionization, a second laser pulse called the probe pulse ionizes the atom. The photoionization signal is measured as a function of the delay time $t$ between the pump and probe signals. The transition probability for absorbing the second photon is greatest when the wave packet is near the core and falls to zero as the wave packet moves away from the nucleus. As a result, the periodicity in the photoionization signal corresponds to the periodicity in the probability for the wave packet to return to its initial configuration. Experiments using this method have detected the initial periodic motion of the wave packet with period $T_{\mathrm{cl}}$, as well as fractional revivals at delay times equal to fractions of $t_{\mathrm{rev}}$, and a full revival at $t_{\mathrm{rev}}$. The fractional revivals are characterized by periodicities in the photoionization signals equal to rational fractions of $T_{\mathrm{cl}}$, as expected.

A second type of pump-probe experiment is based on Ramsey's method of separated oscillating fields. ${ }^{41}$ In this method, an initial laser pulse creates a wave packet, which is then excited by a second identical laser pulse. Depending on the relative phase between the two time-separated optical pulses, the upper-state population in the wave packet can be either increased or reduced by the second pulse. This population transfer between the excited levels and the ground state falls to zero as the wave packet moves towards its outer turning point. The population of the excited levels as a function of the delay time thus appears as a rapidly oscillating function, due to the Ramsey interference, that is modulated by an envelope dependent on the wave-packet motion. By monitoring the population of the excited levels as a function of time using electric-field ionization, the motion of the wave packet can be detected via the periodicities in the envelope function. Since electric-field ionization is more efficient than photoionization, the Ramsey method is better able to resolve fractional revivals. Indeed, using this method, fractional revivals consisting of as many as seven subsidiary wave packets have been detected. ${ }^{37}$

The wave function for a Rydberg wave packet can be written as a superposition of energy eigenstates

$$
\Psi(\vec{r}, t)=\sum_{n} c_{n} \psi_{n}(\vec{r}) e^{-i E_{n} t / \hbar},
$$

where $c_{n}$ are weighting coefficients and $\psi_{n}$ are energy eigenstates. For excitations with a short laser pulse, the coefficients $c_{n}$ are strongly peaked around a central value of $n$ equal to $\bar{n}$. This permits a Taylor expansion of the energy around the value $\bar{n}$. The first three derivative terms in this expansion define the time scales $T_{\mathrm{cl}}, t_{\mathrm{rev}}$, and $t_{\mathrm{sr}}$ as follows:

$$
\begin{gathered}
T_{\mathrm{cl}}=\frac{2 \pi}{\left|\left(E_{\bar{n}}\right)^{\prime}\right|}, \\
t_{\mathrm{rev}}=\frac{2 \pi}{\frac{1}{2}\left|\left(E_{\bar{n}}\right)^{\prime \prime}\right|}, \\
t_{\mathrm{sr}}=\frac{2 \pi}{\frac{1}{6}\left|\left(E_{\bar{n}}\right)^{\prime \prime \prime}\right|} .
\end{gathered}
$$

The primes denote derivatives with respect to $n$. The evolution of the wave packet is controlled by the interplay between these three time scales in the time-dependent phase.

For short times $t \ll t_{\text {rev }}$, only the first-derivative term in the Taylor expansion of $E_{n}$ contributes significantly, and we can write $\psi(\vec{r}, t) \approx \psi_{\mathrm{cl}}(\vec{r}, t)$, where

$$
\psi_{\mathrm{cl}}(\vec{r}, t)=\sum_{n} c_{n} \psi_{n}(\vec{r}) e^{-i 2 \pi(n-\bar{n}) t / T_{\mathrm{cl}}}
$$

and we have omitted an overall phase. The function $\psi_{\mathrm{cl}}$ is periodic with period $T_{\mathrm{cl}}$. This expression is valid only for $t \ll t_{\text {rev }}$, however. Eventually the second-derivative term in the phase becomes appreciable, and the initial periodic motion is destroyed. The fractional revivals occur at times 
$t=m t_{\text {rev }} / n$, where $m$ and $n$ are relatively prime integers $(m \leqslant n)$. At these times, it has been shown ${ }^{30}$ that, with third and higher derivatives neglected, the wave function can be written as a sum of subsidiary wave functions:

$$
\Psi(\vec{r}, t) \approx \sum_{s=0}^{l-1} a_{s} \psi_{\mathrm{cl}}\left(\vec{r}, t+\frac{s}{l} T_{\mathrm{cl}}\right) .
$$

The coefficients $a_{s}$ and the integers $l$ depend on $m$ and $n$ and are defined in Ref. 30. The moduli of the $a_{s}$ are equal for all $l$, which means that the terms in the sum are equally weighted. The form of this equation shows that at the fractional revivals, the wave function equals a sum of subsidiary waves, each of which has the form of the initial wave function but is shifted in its orbit by a fraction of $T_{\mathrm{cl}}$.

The periodicities in the motion of the wave packet are most easily studied using the autocorrelation function ${ }^{31}$ $A(t)=\langle\Psi(\vec{r}, 0) \mid \Psi(\vec{r}, t)\rangle$. The absolute square of the autocorrelation function as a function of time gives the probability for the wave packet to return to its initial configuration. In a pump-probe experiment using photoionization, the periodicities in the photoionization signal should match those in $|A(t)|^{2}$, since both measure the probability for the wave packet to return to the core. Alternatively, in pump-probe experiments using the Ramsey method, the ionization signal depends on the real part of the autocorrelation function $\operatorname{Re}\{A(t)\}=\operatorname{Re}\{\langle\Psi(\vec{r}, 0) \mid \Psi(\vec{r}, t)\rangle\}$. This highly oscillatory function is modulated by an envelope that depends on the wave-packet motion. In the following section, an analysis of the revival structure of edge-magnetoplasmon wave packets is presented and experimental detection methods are discussed.

\section{DYNAMICS OF EMP'S: THE EDGE-MAGNETOPLASMON WAVE PACKET}

The calculations in this section are based on the microscopic theory of QH samples with sharp edges and $\nu=1 / \mathrm{m}$. In this case, the edges have a single branch of chiral bosons. The calculations could readily be generalized to cases where the microscopic physics of the edge requires more elaborate boson models, if motivated by future experimental advances. For a schematic picture of the experimental geometry we have in mind, see Fig. 1. We show that the edge of quantumHall systems can be prepared in a state which is a superposition of EMP's narrowly distributed around a peak mode number. An exact expression for the time evolution of these wave packets can be obtained and these show an intricate revival structure. Numerical calculations for a particular set of model parameters are used to illustrate the latter.

\section{A. Preparation and evolution of an EMP WP}

We consider a $\mathrm{QH}$ sample subject to a time-dependent external potential which couples to the charge density of the system. We are motivated in large part by an experiment performed by Ashoori et al., ${ }^{21}$ which used capacitive coupling between the edge charge density and gates close to the edge of the QH sample to create EMP excitations and to detect their presence. The Hamiltonian describing this coupling is

$$
H=H_{0}+V^{\text {ext }}(t),
$$

with the unperturbed time-independent Hamiltonian for free bosons (EMP's) given by

$$
H_{0}=\sum_{M>0} \varepsilon_{M}^{C} b_{M}^{\dagger} b_{M},
$$

and the time-dependent external potential

$$
\begin{aligned}
V^{\mathrm{ext}}(t) & =u(t) R \int_{0}^{2 \pi} d \theta V^{\mathrm{ext}}(\theta) \varrho(\theta) \\
& =u(t) \sum_{M>0}(\nu M)^{1 / 2}\left[V_{-M}^{\mathrm{ext}} b_{M}+V_{M}^{\mathrm{ext}} b_{M}^{\dagger}\right] .
\end{aligned}
$$

Here, $u(t)$ is the time-dependent voltage pulse applied to the exciting gate; see Fig. 1. The angle $\theta$ parametrizes the coordinate along the edge. The quantity $\varrho(\theta)$ is the $1 \mathrm{D}$ electron density along the edge and $\varrho_{M}$ is its Fourier transform, while $V^{\text {ext }}(\theta)\left(V_{M}^{\text {ext }}\right)$ models (the Fourier transform of) the coupling between the gate and the 1D density along the edge, and $\nu$ is the filling factor. Equation (2) was used to obtain Eq. (9b). We have in mind the situation where the vertical separation between the plane containing the exciting gate and the plane containing the 2D electron layer is much smaller than the transverse size of the gate so that $V^{\mathrm{ext}}(\theta) \approx 1$ for the portion of the edge under the gate and smoothly falls to zero outside this region. The time evolution of the system is calculated most straightforwardly in the Heisenberg picture where the operators carry all the time dependence and the states are time independent:

$$
b_{M}^{ \pm}(t):=e^{i H t} b_{M}^{ \pm} e^{-i H t} .
$$

(Factors of $\hbar$ are absorbed in our quantum-Hall units.) The explicit form of these operators may be obtained by solving the Heisenberg equation of motion. In order to compress the notation, we write simultaneous equations for creation and annihilation operators; note that $b_{M}^{+} \equiv b_{M}^{\dagger}$ and $b_{M}^{-} \equiv b_{M}$. The Heisenberg equation of motion then reads

$$
\begin{aligned}
i \partial_{t} b_{M}^{ \pm}(t) & =\left[b_{M}^{ \pm}(t), H\right] \\
& =\mp \varepsilon_{M}^{C} b_{M}^{ \pm}(t) \mp(\nu M)^{1 / 2} u(t) V_{\mp M}^{\mathrm{ext}},
\end{aligned}
$$

where the second line follows from the first using the commutation relations for bosonic operators with the Hamiltonian. The solution is

$$
b_{M}^{ \pm}(t)=\left[b_{M}^{ \pm}+B_{\mp M}(t)\right] \exp \left[ \pm i \varepsilon_{M}^{C} t\right],
$$

where $B_{ \pm M}(t)$ are complex numbers:

$$
B_{ \pm M}(t)=(\nu M)^{1 / 2} V_{ \pm M}^{\mathrm{ext}}(\mp i) \int_{-\infty}^{t} d \tau u(\tau) \exp \left[ \pm i \varepsilon_{M}^{C} \tau\right]
$$

The undisturbed edge is a collection of independent harmonic oscillators. When the edge is disturbed by an external potential which couples to the edge charge density, each harmonic oscillator is subject to a different time-dependent external force. Equation (12) implies that when the edge is 
initially in its ground state, the effect of the external force is to put each oscillator in a coherent state described by the complex field $B_{ \pm M}(t)$.

Inserting Eq. (12) into the expression for the electron number density along the edge [Eq. (2)], we obtain the Heisenberg-picture expression for the edge density operator:

$$
\varrho(\theta, t)=\varrho_{0}(\theta, t)+\sigma(\theta, t)
$$

where

$$
\begin{aligned}
\varrho_{0}(\theta, t)= & \sum_{M>0} \frac{(\nu M)^{1 / 2}}{2 \pi R}\left\{b_{M} \exp \left[i\left(M \theta-\varepsilon_{M}^{C} t\right)\right]\right. \\
& \left.+b_{M}^{\dagger} \exp \left[-i\left(M \theta-\varepsilon_{M}^{C} t\right)\right]\right\} \\
\sigma(\theta, t)= & \sum_{M>0} \frac{(\nu M)^{1 / 2}}{2 \pi R}\left\{B_{M}(t) \exp \left[i\left(M \theta-\varepsilon_{M}^{C} t\right)\right]\right. \\
& \left.+B_{-M}(t) \exp \left[-i\left(M \theta-\varepsilon_{M}^{C} t\right)\right]\right\} .
\end{aligned}
$$

Equation (14a) is an exact expression capturing the impact of the external time-dependent potential $V^{\text {ext }}(t)$ on the edge charge density. Note that the effect of $V^{\text {ext }}(t)$ shows up only in Eq. (14c) for $\sigma(\theta, t)$, and that $\varrho_{0}(\theta, t)$ does not contribute to the expectation value of $\varrho(\theta, t)$ since it does not conserve boson occupation numbers. As a result the density response to an external potential is temperature independent.

In the nonequilibrium state created by the excitation pulse, the time-dependent charge density, given by Eq. (14a) $[\langle\varrho(\theta, t)\rangle=\sigma(\theta, t)]$, is identical to the time-dependent charge density of a linear combination of classical normal modes. We will henceforth refer to this quantum state of the edge as an edge-magnetoplasmon wave-packet (EMP WP) state. The time-evolution of the wave packet is given by Eq. (14c). As was discussed above, the spatial structure of the prepared wave packet as well as its evolution after switching off the potential is the same at any temperature.

It is shown in Appendix A that it is possible to create a wave packet that has mode numbers strongly peaked around a central value $\widetilde{M}$. One possible scenario uses a sequence of short voltage pulses on the gate to excite a superposition of eigenmodes, in analogy to the use of a short laser pulse to excite a superposition of Rydberg states. Using this method of excitation, it should be possible to produce wave packets with a mean mode number $\widetilde{M} \approx 10-100$.

To detect the wave packet, a second (detecting) gate can be located at coordinate $\theta_{0}$ relative to the exciting gate. (Alternately, the exciting gate could also serve as the detecting gate.) The charge signal at the detecting gate can be computed as a function of the delay time $t$ :

$$
Q(t)=R \int_{0}^{2 \pi} d \theta V^{\operatorname{det}}\left(\theta-\theta_{0}\right)\langle\varrho(\theta, t)\rangle,
$$

where $V^{\operatorname{det}}(\theta) \approx 1$ under the gate and smoothly goes to zero outside the gates because of fringe fields. We can loosely think of $Q(t)$ as the charge under the gate. The voltage signal on the gate should then be determined by the effective gate capacitance. (Note that we are not accounting for changes in the effective interaction between electrons in the 2D ES's due to screening charges on the gates.) In the ex- periments of Ref. 21 the voltage signal is approximately 1 $\mu \mathrm{V}$ per electron under the gate. [If the same gate were used for excitation and detection we would have $V^{\operatorname{det}}(\theta)=V^{\text {ext }}(\theta)$.] Inserting Eq. (14a) into Eq. (15), we obtain for $Q(t)$

$$
Q(t)=2 \operatorname{Re}\left\{\frac{1}{2 \pi R} \sum_{M>0} Q_{M}(t) \exp \left[i\left(M \theta_{0}-\varepsilon_{M}^{C} t\right)\right]\right\}
$$

with the Fourier components given by

$$
\begin{aligned}
Q_{M}(t) & =(\nu M)^{1 / 2} B_{M}(t) V_{-M}^{\operatorname{det}} \\
& =\nu M V_{M}^{\mathrm{ext}} V_{-M}^{\mathrm{det}}(-i) \int_{-\infty}^{t} d \tau u(\tau) \exp \left[i \varepsilon_{M}^{C} \tau\right] .
\end{aligned}
$$

This method of creating an EMP WP using a time-dependent gate voltage and detecting the voltage pulse induced at a second gate by the time-dependent charge density of the propagating EMP WP is partially analogous to the phasesensitive Ramsey method of detection for Rydberg wave packets. ${ }^{42}$

We will refer to the picture of the gate-characteristic functions $V^{\text {ext }}(\theta)$ and $V^{\operatorname{det}}(\theta)$ explained above, in which the gate is most sensitive to charges that are located in its immediate vicinity, as the local-capacitor model. For the calculations reported below we take $V^{\text {ext }}(\theta)=V^{\operatorname{det}}(\theta)=\exp \left[-\left(\theta R / \zeta_{G}\right)^{2}\right]$, where $\zeta_{G}$ is the size of the gate. For the Fourier transforms we find that $V_{M}^{\mathrm{ext}}=V_{M}^{\mathrm{det}} \sim\left(\zeta_{G} / R\right) \exp \left[-\left(M \zeta_{G} / R\right)^{2}\right]$. The factor $V_{M}^{\text {ext }} V_{-M}^{\text {det }}$ in Eq. (17b) then precludes the observation of EMP modes with the wave number $M>R / \zeta_{G}$. This fact leads to an important constraint on the observability of an EMP WP. If the excitation scheme (as discussed in detail in Appendix A) creates an initial wave packet that is a superposition of EMP modes with the dominant contribution from the mode with wave number $\widetilde{M} \gg 1$, then optimal observability of this wave packet using the charge signal $Q(t)$ requires $\widetilde{M} \zeta_{G} / R<1$.

\section{B. Revival structure}

To examine the revival structure of an EMP WP, we first consider the expectation value for the electron number density along the edge. We assume that the excitation pulse is turned off at time $\tau$. Then for $t>\tau$

$$
\langle\varrho(\theta, t)\rangle=2 \operatorname{Re}\left\{\sum_{M>0} c_{M} e^{i M \theta} e^{-i \varepsilon_{M}^{C} t}\right\},
$$

where

$$
c_{M}=\frac{\nu M}{2 \pi R} V_{M}^{\mathrm{ext}}\left(-i \int_{-\infty}^{\tau} d \tau^{\prime} u\left(\tau^{\prime}\right) \exp \left[i \varepsilon_{M}^{C} \tau^{\prime}\right]\right) .
$$

The coefficients $c_{M}$ act as weighting functions for the different modes $M$. Figure 2 illustrates some possible distributions for the weightings in $M$ resulting from excitation sequences detailed in Appendix A. As a result of the weighting distribution, only those energies $\varepsilon_{M}^{C}$ in Eq. (18) near $\varepsilon_{\widetilde{M}}^{C}$ will 


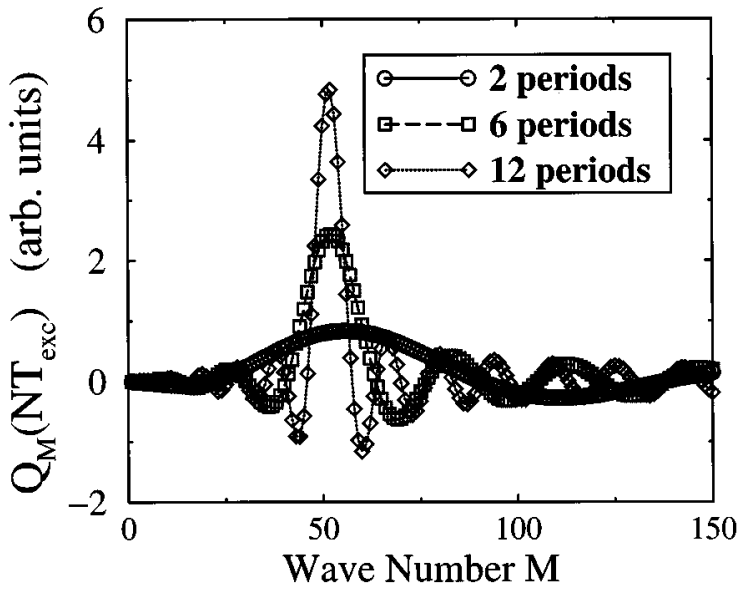

FIG. 2. Envelope function $Q_{M}\left(N T_{\text {exc }}\right)$ for the wave packet as detected at the end of $N$ excitation pulses of duration $T_{\text {exc }}$. [See Eqs. (16) and (17) for the definition of the envelope.] The wave packet is created by multiple short excitation pulses as discussed in Appendix A 2. These curves were all calculated for $T_{\text {exc }}=0.005 \times 2 \pi R$ and $R=2500$ in quantum-Hall units. The figure shows results for different numbers of pulses $N$. The wave number $\widetilde{M}$ with a maximal weight is determined by $T_{\mathrm{exc}}$. The larger $N$, the sharper the peak in the envelope function at $M=\widetilde{M}$. For simplicity, it is assumed that the geometry of the gates does not influence the envelope function for the range of wave numbers shown.

contribute to the sum. This permits a Taylor expansion of $\varepsilon_{M}^{C}$ around the central value $\varepsilon_{\widetilde{M}}^{C}$.

The derivative terms in this expansion define the time scales that control the evolution and revival structure of the wave packet, as discussed above in the section on Rydberg wave packets. In this case the expressions for the time scales are [cf. Eqs. (4)]

$$
\begin{gathered}
T_{\mathrm{cl}}=\frac{2 \pi}{\left|\left(\varepsilon_{\widetilde{M}}^{C}\right)^{\prime}\right|}=\frac{2 \pi R}{\left(\ln \frac{R}{\alpha \widetilde{M}}-1\right)}, \\
t_{\mathrm{rev}}=\frac{2 \pi}{\frac{1}{2}\left|\left(\varepsilon_{\widetilde{M}}^{C}\right)^{\prime \prime}\right|}=4 \pi R \tilde{M}, \\
t_{\mathrm{sr}}=\frac{2 \pi}{\frac{1}{6}\left|\left(\varepsilon_{\widetilde{M}}^{C}\right)^{\prime \prime \prime}\right|}=12 \pi R \widetilde{M}^{2} .
\end{gathered}
$$

The primes denote derivatives with respect to $M$. Equations (20) define the classical orbit period, the revival time, and the super revival time, respectively, for EMP WP's.

For large values of $\widetilde{M} \approx 10-100$ and typical values of $R \approx 10^{4}$, we find $t_{\mathrm{sr}} \gg t_{\mathrm{rev}} \gg T_{\mathrm{cl}} \gg T_{\mathrm{ph}}:=2 \pi / \varepsilon_{\widetilde{M}}^{C}$. Therefore, for times $t \ll t_{\text {rev }}$, we can approximate $\langle\varrho(\theta, t)\rangle \approx 2 \operatorname{Re}\left\{\widetilde{\varrho}_{\mathrm{cl}}(\theta, t)\right\}$, where

$$
\begin{aligned}
\widetilde{\varrho}_{\mathrm{cl}}(\theta, t) & =e^{i\left(\tilde{M} \theta-2 \pi t / T_{\mathrm{ph}}\right)} \sum_{M>0} c_{M} e^{i(M-\widetilde{M})\left(\theta-2 \pi t / T_{\mathrm{cl}}\right)} \\
& =e^{i\left(\tilde{M} \theta-2 \pi t / T_{\mathrm{ph}}\right)} \cdot \varrho_{\mathrm{cl}}(\theta, t)
\end{aligned}
$$

Here $T_{\mathrm{ph}}=2 \pi R /(\tilde{M} \ln [R /(\alpha \tilde{M})])$. Thus $\widetilde{\varrho}_{\mathrm{cl}}(\theta, t)$ is the product of a rapidly oscillating ${ }^{43}$ (both in space and time) phase factor and a more slowly varying periodic envelope function. The period of the rapid spatial oscillations is $2 \pi / \widetilde{M}$, whereas $T_{\mathrm{ph}}$ is the period of the rapid temporal oscillations. If the additional phase factor were not present, the charge density in this approximation would circulate around the edge without distortion and with period $T_{\mathrm{cl}}$. This motion resembles the classical drift motion ${ }^{44}$ of the cyclotron orbit of a charged particle in a strong magnetic field; it is perpendicular to both magnetic and electric fields and has a speed $v_{\mathrm{dr}}=c E / B$. In the present case, the electric field is perpendicular to the edge of the 2D ES so that the drift is along the edge and the classical period is ${ }^{45} T_{\mathrm{cl}} \approx 2 \pi R / v_{\mathrm{dr}}$. The electric field which yields our classical orbit period is that from the neutralizing background required to stabilize a macroscopic system of charged particles. ${ }^{45}$ Because of the additional phase factor this classical motion appears as the envelope of a more rapid oscillation of edge charge density. In what follows, we focus on the evolution of this classical envelope function at longer times.

For times greater than $T_{\mathrm{cl}}$, the second-derivative term will eventually contribute to the time-dependent phase, and we expect a revival structure analogous to the fractional and full revivals of Rydberg wave packets. Indeed, at the times $t=m t_{\text {rev }} / n$, with $m$ and $n$ relatively prime $(m \leqslant n)$, we can write the sum over $M$ [in Eq. (18)] as a sum of subsidiary functions,

$$
\begin{aligned}
\langle\varrho(\theta, t)\rangle \approx & 2 \operatorname{Re}\left\{e^{i\left(\tilde{M} \theta-2 \pi t / T_{\mathrm{ph}}\right)}\right. \\
& \left.\times \sum_{s=0}^{l-1} a_{s} \varrho_{\mathrm{cl}}\left(\theta, t+\frac{s}{l} T_{\mathrm{cl}}\right)\right\} .
\end{aligned}
$$

The coefficients $a_{s}$ are given by

$$
a_{s}=\frac{1}{l} \sum_{M^{\prime}=0}^{l-1} \exp \left[2 \pi i \frac{m}{n}\left(M^{\prime}\right)^{2}\right] \exp \left[2 \pi i M^{\prime} \frac{s}{l}\right],
$$

with

$$
l= \begin{cases}\frac{n}{2} & \text { if } n=0(\bmod 4) \\ n & \text { if } n \neq 0(\bmod 4) .\end{cases}
$$

The moduli $\left|a_{s}\right|$ are equal for all $l$. Therefore, the sum over $s$ in Eq. (22) consists of an equally weighted sum of subsidiary functions that are shifted in time by fractions of the classical period $T_{\mathrm{cl}}$. This demonstrates that at the fractional revivals the EMP WP's can be written as a sum of subsidiary waves. The motion of the wave packet is periodic with a period equal to a fraction of $T_{\mathrm{cl}}$. The full revival occurs when $m=n=1$. In this case $l=1, a_{0}=1$ is the only nonzero coefficient in Eq. (22), and the wave-packet sum consists of a single term which has the same form as the initial wave packet. This analysis for $t \leqslant t_{\text {rev }}$ ignores contributions from the higher-order terms in the phase. As a result of these higher-order terms, the full revival is not a perfect revival in that it does not exactly equal the initial wave packet. Including the third-order term in the time-dependent phase, which 

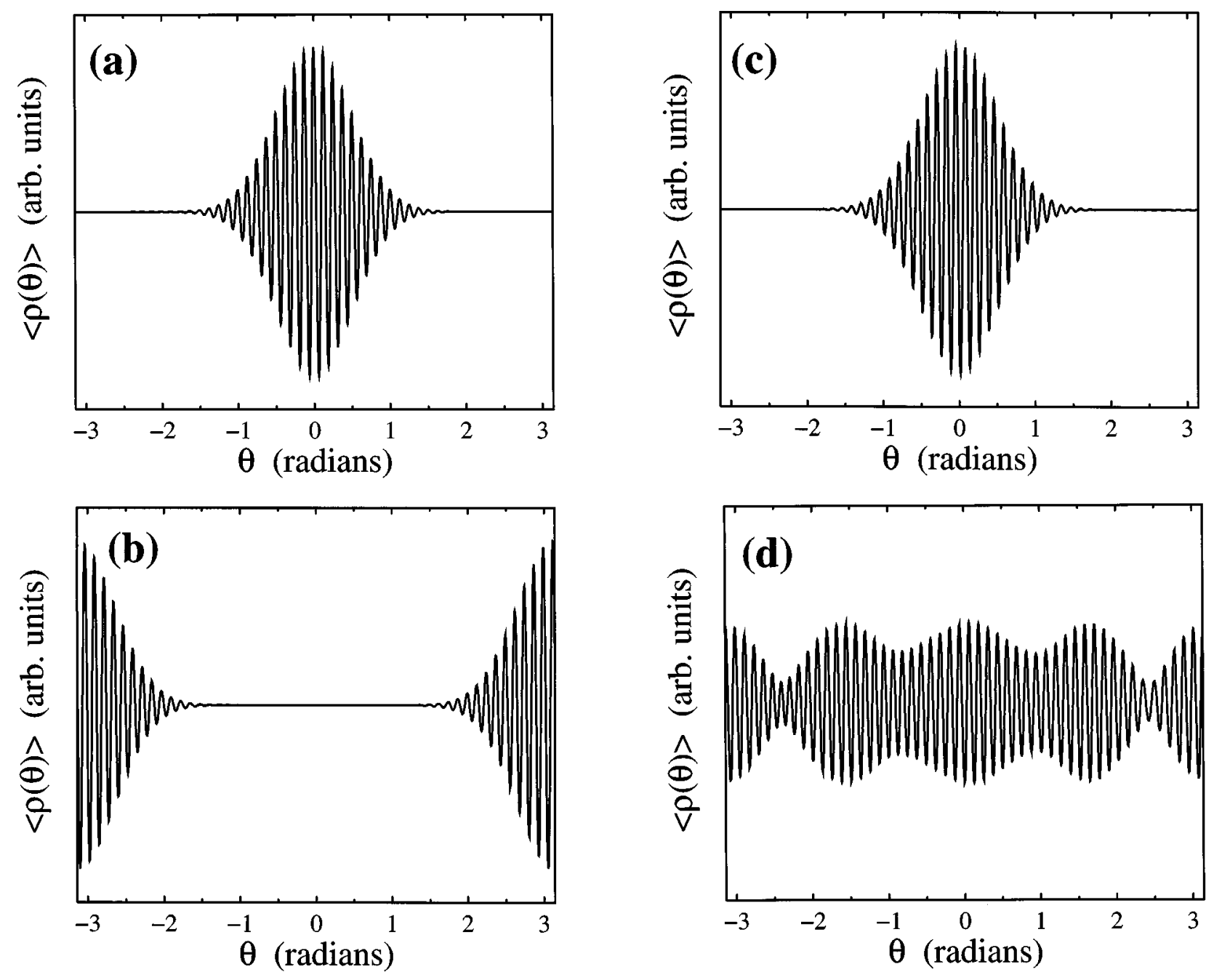

FIG. 3. The unnormalized number density $\langle\rho(\theta, t)\rangle$ is plotted as a function of angle $\theta$ at early times. (a) $t=0$, (b) $t=T_{\mathrm{cl}} / 2$, (c) $t=T_{\mathrm{cl}}$, and (d) $t=50 T_{\mathrm{cl}}$.

depends on the time scale $t_{\mathrm{sr}}$, would lead to an analysis of the super revival structure analogous to that performed previously for Rydberg wave packets.

The experimentally measured quantity is not $\varrho(\theta, t)$ but the charge signal $Q(t)$ at the detecting gate defined in Eq. (16). In this expression the weighting coefficients $Q_{M}(t)$ are constant after the excitation pulse has been turned off. Expanding $\varepsilon_{M}^{C}$ in a Taylor series in $M$ around the value $\tilde{M}$ shows that $Q(t)$ has a time dependence similar to $\langle\varrho(\theta, t)\rangle$. The full and fractional periodicities in $T_{\mathrm{cl}}$ should therefore be exhibited in the time dependence of the charge signal $Q(t)$.

\section{Example}

As an example, we consider an EMP WP with $\widetilde{M}=50$. In the next section, we discuss the experimental feasibility of creating and observing a wave packet with mode numbers in this range. In realistic samples, such a wave packet will be damped and lose phase coherence due to electron-phonon interactions, and possibly also due to interactions with electrons in the bulk of the two-dimensional system when it has localized low-energy excitations. In this section, we ignore for the moment damping and loss of phase coherence of the wave packet and examine the resulting idealized revival structure for times up to $t_{\text {rev }}$.

As shown in Appendix A, there are many possible weighting distributions for the coefficients $c_{M}$, depending on the experimental configuration. The analysis of the revivals given above requires only that the coefficients $c_{M}$ be strongly peaked around a central value $\tilde{M}$. For the sake of definiteness we take a particular example in this subsection, modeling the coefficients $c_{M}$ by a Gaussian distribution centered on $\widetilde{M}=50$ with width $\sigma_{M}=2$. We also set $\alpha=1$ and $R=10^{4}$. From Table I for typical magnetic-field strengths this corresponds to a sample with linear dimension $\sim 100$ $\mu \mathrm{m}$ and time scales starting from the nanosecond range. To be specific, we use $\nu=1$ and $B=10 \mathrm{~T}$, which fixes our time scales to $T_{\mathrm{cl}} \approx 2.2 \mathrm{~ns}, t_{\mathrm{rev}} \approx 942 \mathrm{~ns}$, and $t_{\mathrm{sr}} \approx 141 \mu \mathrm{s}$. In the chiral-boson model the time evolution is independent of the overall strength of the signal so we plot results for $\langle\varrho(\theta, t)\rangle$ calculated from Eq. (18) as a function of the angle $\theta$ at various times in arbitrary units.

Figure 3(a) shows the initial wave packet for the Gaussian model considered in this section, which sums to a smooth 

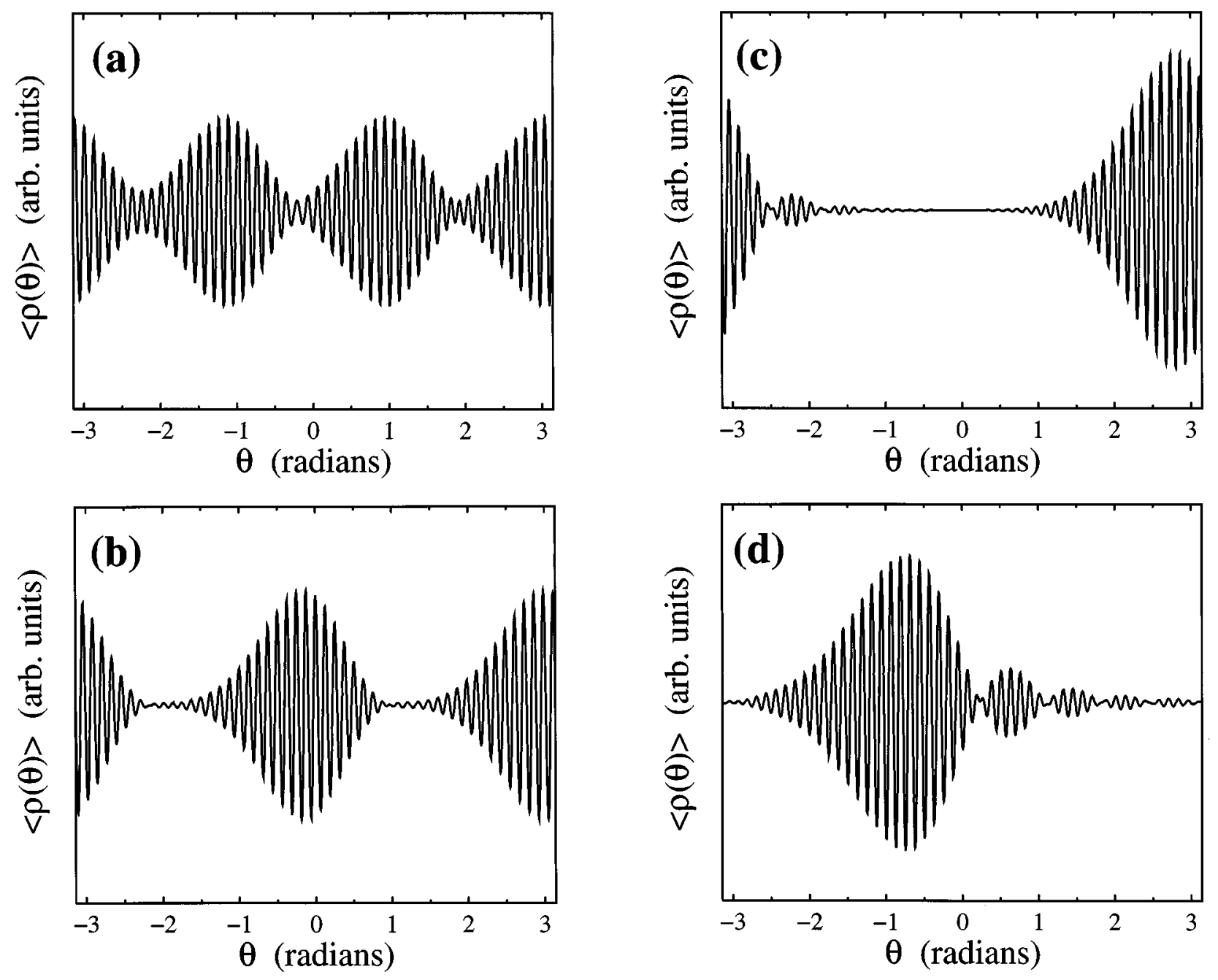

FIG. 4. The unnormalized number density $\langle\rho(\theta, t)\rangle$ as a function of angle $\theta$ at the fractional revivals. (a) $t=t_{\text {rev }} / 6$, (b) $t=t_{\text {rev }} / 4$, (c) $t=t_{\text {rev }} / 2$, and (d) $t=t_{\text {rev }}$.

envelope on an oscillating background. Figures 3(b) and 3(c) show the wave packet at times $T_{\mathrm{cl}} / 2$ and $T_{\mathrm{cl}}$, respectively. It is seen that initially the wave packet maintains its shape and exhibits the classical periodicity. However, for times beyond $T_{\mathrm{cl}}$ quantum interference effects commence. Figure 3(d) shows the wave packet at $50 T_{\mathrm{cl}}$. After 50 classical periods, the wave packet has spread and is no longer localized.

Figure 4 shows the fractional and full revivals. At $t=t_{\text {rev }} / 6$, there are three nonzero terms in the sum in Eq. (22). The wave packet splits into three equally weighted subsidiary waves as illustrated in Fig. 4(a). The motion of the wave packet is periodic with period $T_{\mathrm{cl}} / 3$. Figure $4(\mathrm{~b})$ shows the wave packet at $t_{\mathrm{rev}} / 4$, at which time it consists of a sum of two distinct subsidiary waves. Here, the motion is periodic with period $T_{\mathrm{cl}} / 2$. A full revival first occurs at $t_{\mathrm{rev}} / 2$. Figure 4(c) shows that a single wave packet has formed. This revival has the quantum-mechanical characteristic of being one-half cycle out of phase with the classical motion. ${ }^{30}$ Only at the time $t_{\text {rev }}$ does a single wave-packet form that is in step with the corresponding classical periodic motion. The full revival at $t_{\text {rev }}$ is shown in Fig. 4(d). It evidently does not exactly match the initial wave packet. A smaller subsidiary wave packet has also formed.
The revival structure as a function of time can also be observed by computing the charge signal $Q(t)$. This is the observable that would be measured in an experiment. We evaluate Eq. (16), using the same Gaussian weighting as above and ignoring the overall normalization.

Figures 5(a) and 5(b) show $Q(t)$ as a function of time for times up to and just beyond $t_{\text {rev }} / 2$. Here, the revival structure is revealed through the periodicity of $Q(t)$. In Fig. 5(a), the initial motion is clearly periodic with period $T_{\mathrm{cl}} \approx 2.2 \mathrm{~ns}$. However, as the wave packet spreads and collapses, the peaks in $Q(t)$ disappear and fractional revivals start to emerge. The peaks at $t_{\text {rev }} / 4 \approx 235 \mathrm{~ns}$ have half the amplitude and periodicity of the initial peaks. This is because the wave packet has reformed into two distinct parts. The peaks at $t_{\text {rev }} / 6 \approx 157$ ns have period $T_{\mathrm{cl}} / 3$ and an even smaller amplitude, corresponding to the formation of three subsidiary wave packets. Figure 5(b) shows the full revival at $t_{\text {rev }} / 2 \approx 471 \mathrm{~ns}$, which is one-half cycle out of phase with the classical motion. Here, the amplitude of the peaks matches that of the initial peaks, and the period is again $T_{\mathrm{cl}}$.

Figure 6 enlarges some of the regions in Fig. 5. Figure 6(a) shows the first few classical cycles for times up to $5 \mathrm{~ns}$. Evidently, the individual peaks with period $T_{\mathrm{cl}}$ actually con- 

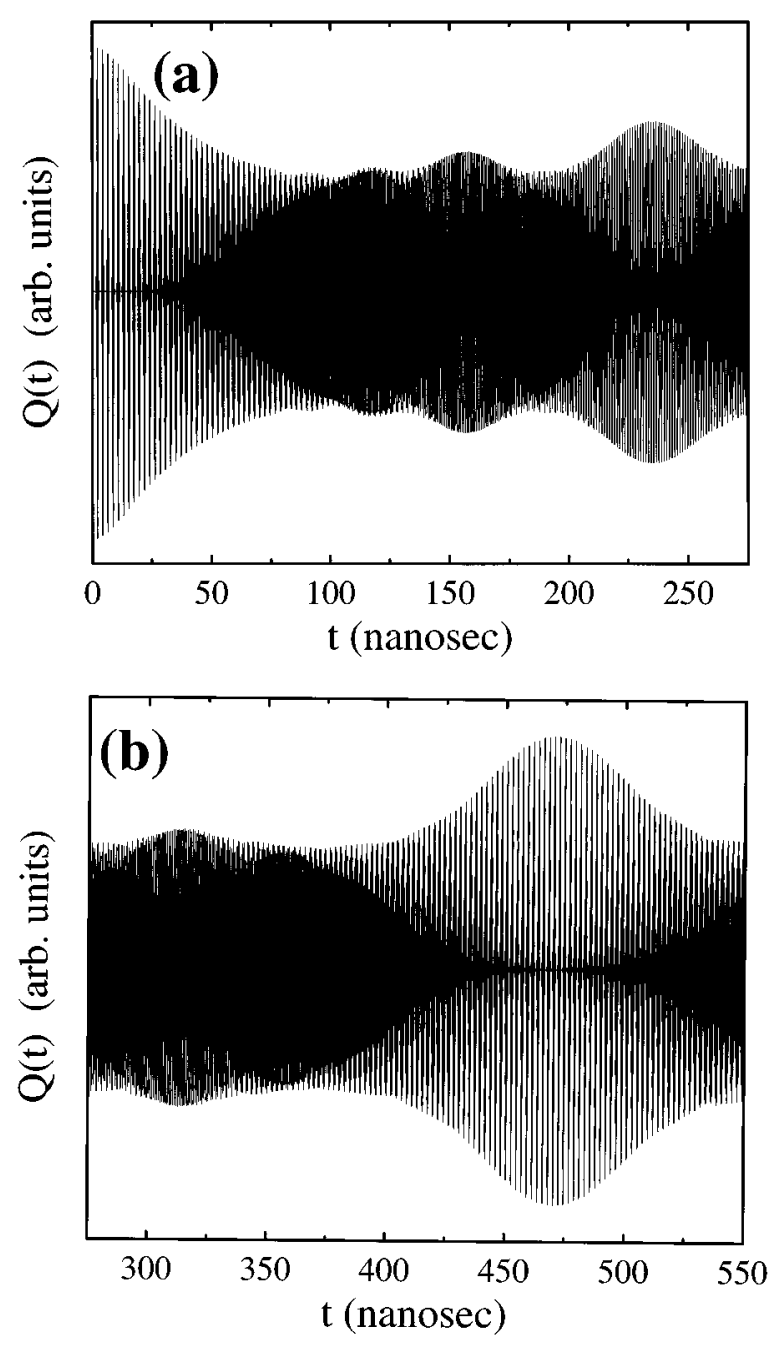

FIG. 5. The charge signal $Q(t)$ is plotted as a function of time in nanoseconds. (a) and (b) illustrate the large-scale periodicities in $Q(t)$ up to times of an order of $t_{\text {rev }} / 2 \approx 471 \mathrm{~ns}$. The rapidly oscillating part of the signal is not resolved in these figures and the individual peaks at short times are separated by the classical period. At partial revivals the period of the revived wave packet is shorter than the classical period. These shorter periods are not resolved in this figure resulting in nearly solid portions of the figure. In this case the extent of the solid region reflects the amplitude of the revived wave packet.

sist of envelopes of highly oscillatory signals. ${ }^{43,42}$ These rapid oscillations cannot be resolved in Fig. 5. The period of the rapid oscillations is $T_{\mathrm{ph}} \approx 0.036 \mathrm{~ns}$. Figure 6(b) shows an enlargement of $Q(t)$ near $t_{\mathrm{rev}} / 6$. The period of the envelope peaks is $T_{\mathrm{cl}} / 3 \approx 0.73 \mathrm{~ns}$. Figure $6(\mathrm{c})$ shows the charge signal near $t_{\text {rev }} / 4$, where two wave packets are moving with period $T_{\mathrm{cl}} / 2 \approx 1.1 \mathrm{~ns}$. The charge signal near $t_{\mathrm{rev}} / 2$ is shown in Fig. 6(d). The period is again $T_{\mathrm{cl}}$, but some distortion of the signal is evident in comparison to the initial peaks in Fig. 6(a).

This example demonstrates explicitly the formation of full and fractional revivals of EMP WP's. The generic features are the same as for Rydberg wave packets. At the fractional revivals, the EMP WP is equal to a sum of subsidiary wave packets that move with a periodicity equal to a fraction of $T_{\mathrm{cl}}$. The semiclassical behavior as well as the revivial struc- ture of EMP WP's can be detected experimentally by measuring periodicities in the envelope that modulates rapid oscillations in the charge signal $Q(t)$. These oscillations are the analog of the Ramsey fringes in Rydberg-wave-packet experiments which use the phase-sensitive method of detection.

\section{Fluctuations: Quantum and thermal}

The EMP WP is a many-mode coherent state: each plasmon mode represents a one-dimensional quantum harmonic oscillator, and the creation scheme described above results in each oscillator mode being excited into a coherent state. The above-mentioned detection scheme yields an average charge signal $Q(t)$ which reflects purely classical behavior of the EMP modes. Quantum fluctuation effects appear only in the noise spectrum of the charge signal.

In our detection scheme, we measure the expectation value of the charge operator $\hat{Q}(t)$ :

$$
\begin{aligned}
\hat{Q}(t) & =R \int_{o}^{2 \pi} d \theta V^{\operatorname{det}}\left(\theta-\theta_{0}\right) \varrho(\theta, t) \\
& =\sum_{M>0}[\nu M]^{1 / 2}\left\{V_{-M}^{\operatorname{det}} b_{M}(t) e^{i M \theta_{0}}+\text { H.c. }\right\} .
\end{aligned}
$$

It is possible to rewrite $\hat{Q}(t)$ as the sum of a term $[=: \delta \hat{Q}(t)]$ that has a vanishing expectation value and contributes only to fluctuations, and a term $[\equiv Q(t)]$ which does not fluctuate at all and equals the expectation value of the charge operator: $\hat{Q}(t)=\delta \hat{Q}(t)+Q(t)$. Explicitly, we find

$$
\delta \hat{Q}(t)=\sum_{M>0}[\nu M]^{1 / 2}\left\{V_{-M}^{\mathrm{det}} b_{M} e^{i\left[M \theta_{0}-\varepsilon_{M}^{C} t\right]}+\text { H.c. }\right\}
$$

and $Q(t)$ was defined in Eq. (16). The variance of the charge signal is readily evaluated:

$$
\begin{gathered}
\left\langle[\Delta \hat{Q}(t)]^{2}\right\rangle=\left\langle[\Delta \hat{Q}(t)]^{2}\right\rangle_{\mathrm{qu}}+\left\langle[\Delta \hat{Q}(t)]^{2}\right\rangle_{\mathrm{th}}, \\
\left\langle[\Delta \hat{Q}(t)]^{2}\right\rangle_{\mathrm{qu}}=\sum_{M>0} \nu M\left|V_{M}^{\mathrm{det}}\right|^{2} \\
\left\langle[\Delta \hat{Q}(t)]^{2}\right\rangle_{\mathrm{th}}=2 \sum_{M>0} \nu M\left|V_{M}^{\mathrm{det}}\right|^{2} n_{M}^{(0)}
\end{gathered}
$$

with $n_{M}^{(0)}$ being the thermal-equilibrium occupation number of the plasmon mode labeled by quantum number $M$. Equations (27b) and (27c) represent the noise due to quantum and thermal fluctuations, respectively.

We assume the detector characteristics to be determined primarily by geometrical properties, such as the dimension $\zeta_{G}$ of the capacitor plate. Within the local-capacitor model, we have $V_{M}^{\text {det }} \sim\left(\zeta_{G} / R\right) \exp \left\{-\left[M \zeta_{G} / R\right]^{2}\right\}$ which yields the result

$$
\left\langle[\Delta \hat{Q}(t)]^{2}\right\rangle \approx \nu \times\left\{\begin{array}{lll}
1 & \text { if } & \zeta_{T}>\zeta_{G} \\
\zeta_{G} / \zeta_{T} & \text { otherwise }
\end{array}\right.
$$



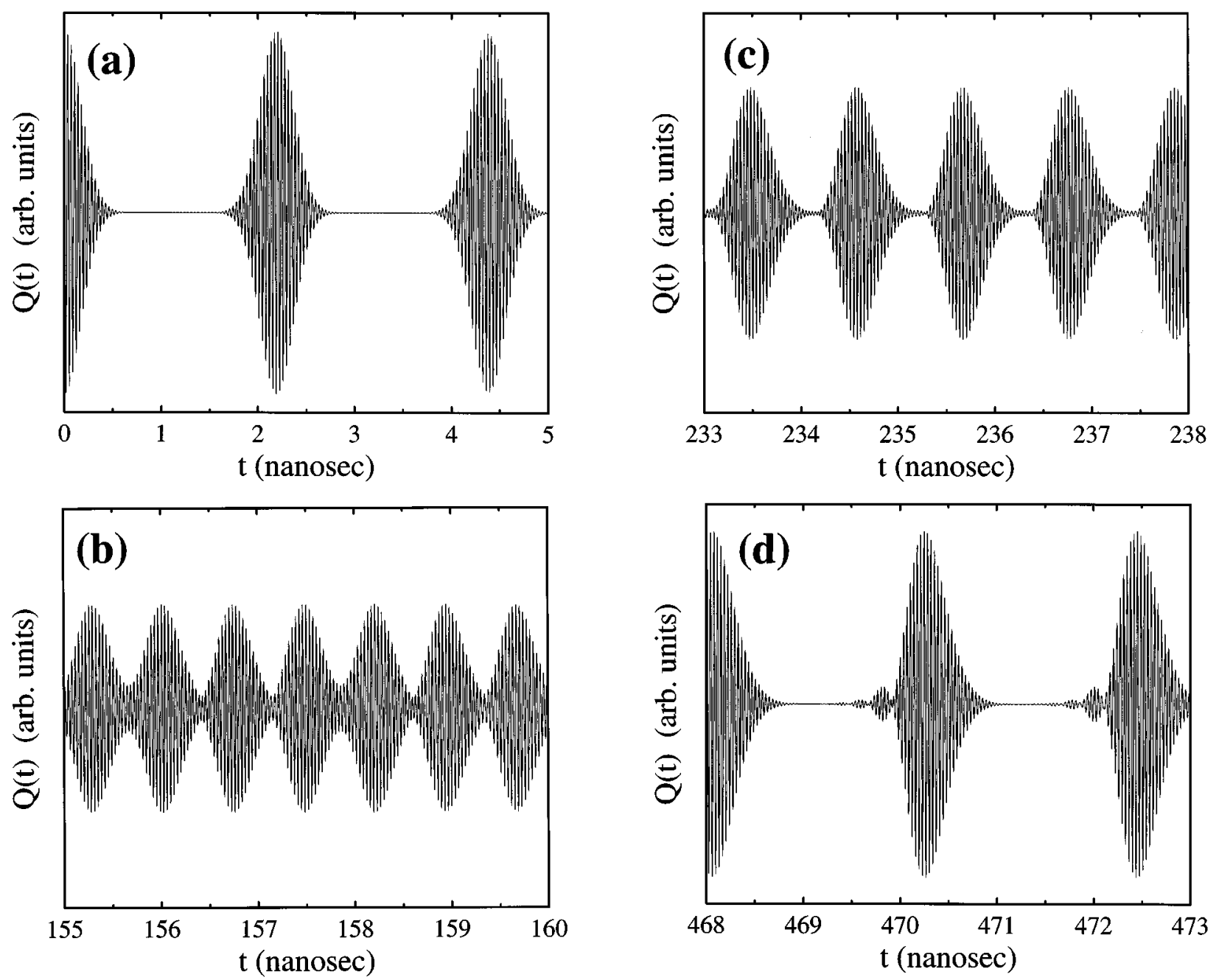

FIG. 6. An enlargement of Fig. 5 at various times. (a) the initial classical cycles for $0 \leqslant t \leqslant 5 \mathrm{~ns}$, revealing the presence of the rapid oscillations in $Q(t)$. (b), (c), and (d) show enlargements of Fig. 5 near the times $t=t_{\text {rev }} / 6, t=t_{\text {rev }} / 4$, and $t=t_{\text {rev }} / 2$, respectively, corresponding to the formation of 3,2 , and 1 subsidiary waves at the fractional revivals.

where the thermal length $\zeta_{T}:=v_{\mathrm{dr}} /\left(\sqrt{4 \pi} k_{B} T\right)$. The regime where $\zeta_{T}>\zeta_{G}$ is dominated by quantum fluctuations, whereas thermal fluctuations are more important if $\zeta_{T}<\zeta_{G}$.

In order to judge the importance of the quantum and thermal fluctuations for experiments detecting the semiclassical behavior and revival structure of EMP WP's, we compare the magnitude of the fluctuations to the amplitude of the charge signal $Q(t)$. As an order-of-magnitude estimate, we find for the case of an EMP WP which was created using the multiple-short-pulse technique (see Appendix):

$$
|Q(t)|^{\max } \sim \nu \frac{\widetilde{M} \zeta_{G}}{R} \frac{u_{0} \zeta_{G}}{v_{\mathrm{dr}}} .
$$

Typical drift velocities $v_{\mathrm{dr}}$ are of the order of $5 \times 10^{5} \mathrm{~m} / \mathrm{s}$, so that we get a numerical estimate

$$
|Q(t)|^{\max } \sim 3 u_{0}[\mathrm{mV}] \zeta_{G}[\mu \mathrm{m}] \times \nu \frac{\widetilde{M} \zeta_{G}}{R} .
$$

Here, $u_{0}$ denotes the amplitude of the voltage pulse which created the EMP WP. Remember that $\widetilde{M} \zeta_{G} / R<1$ is required to enable the detection of the EMP WP using the charge signal. For a signal-to-noise ratio greater than unity, the amplitude of the voltage pulse has to satisfy

$$
u_{0} \gg \frac{R}{\widetilde{M} \zeta_{G}} \frac{v_{\mathrm{dr}}}{\zeta_{G}} \max \left\{1,\left(\zeta_{G} / \zeta_{T}\right)^{1 / 2}\right\}
$$

or based on the numerical estimate above

$$
u_{0}[\mathrm{mV}] \gg \frac{0.3}{\zeta_{G}[\mu \mathrm{m}]} \times \frac{R}{\widetilde{M} \zeta_{G}} .
$$

\section{FINITE LIFETIME OF EDGE-MAGNETOPLASMON WAVE PACKETS}

The previous section analyzed the formation and revival structure of EMP WP's, assuming an infinite lifetime for the EMP's which form the wave packet. It was shown that thermal effects have no influence on the preparation and evolution of these wave packets apart from contributing to fluctuations. The revival structures that we have discussed require the coherent evolution of the EMP system. Coupling to the bulk electron system can in principle destroy phase 
coherence and lead to dissipation for the EMP. However, by tuning the magnetic field into the middle of the $\mathrm{QH}$ plateau, it is possible to virtually eliminate dissipation due to coupling to the bulk: Since the energy of EMP's is much smaller than the charge gap, and the EMP wavelength $(\sim$ system size) then by far exceeds the localization length ( $\sim$ magnetic length in the middle of the QH plateau), it is safe to neglect the response of the bulk to the EMP excitations. To be somewhat more quantitative, we follow the phenomenological analysis in Ref. 12, from which it follows that the damping rate $\Gamma_{\text {bulk }}$ of EMP WP's due to coupling to the bulk is given by the relation

$$
\frac{\Gamma_{\mathrm{bulk}}^{-1}}{T_{\mathrm{cl}}} \sim 2 \frac{\tau^{*}}{T_{\mathrm{cl}}} \ln [R / \widetilde{M}] .
$$

Here $\tau^{*}$ denotes the low-temperature, mid-plateau electron scattering time in the bulk of the 2D ES, which is always extremely long. For example, in a typical 2D ES, the value of $\tau^{*}$ extracted $^{46}$ from experimental data was $\tau^{*} \sim 10^{-3} \mathrm{sec}$. For the values of $T_{\mathrm{cl}}$ considered in this work, we find $\Gamma_{\text {bulk }}^{-1} / T_{\mathrm{cl}} \sim 10^{6} \ln [R / \widetilde{M}]$. The scattering time $\Gamma_{\text {bulk }}^{-1}$ is long compared to the time for EMP-phonon scattering calculated below [see Eq. (39a)].

In realistic systems, electrons in the 2D ES will be coupled to the semiconductor host material via various physical processes. For experimentally realistic temperatures and parameter ranges in semiconductors, the most important process will typically be the coupling of electrons to 3D acoustic phonons. This coupling leads to a finite lifetime for EMP's, which we calculate in this section. Comparing the lifetime to the relevant time scales for semiclassical behavior $\left(T_{\mathrm{cl}}\right)$ and for revivals $\left(t_{\text {rev }}\right)$, we can determine the observability of these effects.

\section{A. Plasmon-phonon coupling-general}

The electron-phonon interaction is specified by the following contribution to the Hamiltonian: ${ }^{47}$

$$
H^{\mathrm{el}-\mathrm{ph}}=\sum_{\vec{q}, \lambda} M^{\lambda}(\vec{q})\left(a_{-\vec{q}, \lambda}^{\dagger}+a_{\vec{q}, \lambda}\right) \varrho_{\vec{q}}^{3 \mathrm{D}},
$$

where the operators $a_{\vec{q}, \lambda}^{\dagger}\left(a_{\vec{q}, \lambda}\right)$ create (annihilate) phonons with $3 \mathrm{D}$ wave vector $\vec{q}$, polarization label $\lambda$, and normalmode frequency $\omega_{\vec{q}}^{\lambda}$. Here, $\varrho_{\vec{q}}^{3 \mathrm{D}}$ denotes the 3D electron density in the QH sample and $M^{\lambda}(\vec{q})$ is a coupling constant whose numerical value is known in most materials of interest. Since EMP's are collective fluctuations of the electron density at the edge of our sample, the electron-phonon interaction leads to an effective coupling between phonons and EMP's. By identifying the contribution to the 3D electron density from EMP's we are able to derive a Hamiltonian which describes the coupling between the EMP and phonon systems. For example, for the disk geometry described in Fig. 1, the Hamiltonian has the form

$$
H^{\mathrm{pl}-\mathrm{ph}}=\sum_{\substack{M>0 \\ \vec{q}, \lambda}} C_{M}^{\lambda}(\vec{q})\left(a_{\vec{q}, \lambda}+a_{-\vec{q}, \lambda}^{\dagger}\right)\left(b_{M} e^{i M \theta_{\vec{q}}}+b_{M}^{\dagger} e^{-i M \theta_{\vec{q}}}\right),
$$

where $\theta_{\vec{q}}$ denotes the azimuthal angle of the wave vector $\vec{q}$ in the plane of the 2D ES's. Details of the derivation of Eq. (35) and an analytic expression for the coupling coefficients $C_{M}^{\lambda}(\vec{q})$ are given in Appendix B. It is possible to derive an expression similar to Eq. (35) which is valid for the strip geometry; this case is also discussed in Appendix B.

To investigate the effect of plasmon-phonon coupling on the evolution of an EMP WP, we consider a single-plasmon Matsubara Green's function defined by

$$
\mathcal{G}_{M}(\tau)=-\left\langle T_{\tau} b_{M}(\tau) b_{M}^{\dagger}(0)\right\rangle,
$$

and the Fourier transform $\mathcal{G}_{M}(\omega)$ of the retarded Green's function, which is obtained from the Fourier transform of $\mathcal{G}_{M}(\tau)$ by a continuation to real frequencies. In the absence of EMP-phonon coupling, $\mathcal{G}_{M}(\omega)$ reduces to the well-known result $^{47}$ for free bosons: $\mathcal{G}_{M}^{0}(\omega)=\left[\omega-\varepsilon_{M}^{\mathrm{C}}+i \delta\right]^{-1}$ reflecting the fact that EMP's are the well-defined excitations of the system. The presence of phonons causes damping (and an energy shift) of the EMP's, leading to the modified result $\mathcal{G}_{M}(\omega)=\left[\omega-\widetilde{\varepsilon_{M}}+i \Gamma_{M} / 2\right]^{-1}$, with $\Gamma_{M}^{-1}$ being the lifetime of the EMP with wave number $M$. We find

$$
\Gamma_{M}=2 \pi \sum_{\vec{q}, \lambda}\left|C_{M}^{\lambda}(\vec{q})\right|^{2} \delta\left(\varepsilon_{M}^{\mathrm{C}}-\omega_{\vec{q}}^{\lambda}\right) .
$$

See Appendix C for details of the calculation.

The meaningful quantity to assess the effect of EMPphonon coupling on the propagation of EMP WP's is $\Gamma_{\tilde{M}}^{-1}$, which we call the lifetime of the EMP WP's. At energy and wave-vector scales appropriate for the observation of EMP WP's, acoustic phonons with dispersion $\omega_{q}^{\lambda}=q c^{\lambda}$ are most important. In a polar semiconductor, both scattering from a deformation potential and piezoelectric effects contribute to the electron-phonon coupling, whereas in a nonpolar semiconductor the piezoelectric part is absent. In the following two subsections, we discuss these two cases separately.

\section{B. Polar semiconductors: GaAs}

At the long wavelengths used to construct EMP WP's, piezoelectric coupling dominates the deformation-potential scattering of electrons. We therefore neglect the contribution from the deformation potential in this subsection. The evaluation of Eq. (37) for the disk case yields

$$
\Gamma_{M} \approx\left(\frac{h_{14}}{\widetilde{c}}\right)^{2} \frac{\epsilon}{\rho} \frac{M}{R}
$$

with $\epsilon$ being the semiconductor bulk dielectric constant, and the piezoelectric coupling constant denoted by $h_{14}$. Here, the quantity $\widetilde{c}$ is of the order of the speed of sound. This estimate is based on the observation that the drift velocity of the EMP WP is typically $\sim 100$ times larger than the speed of sound so that typical projections of the phonon wave vector onto the 2D plane lead to large Bessel-function arguments. (Typical phonon wavelengths are very small compared to the size of the disk.) Note that modes with a higher wave number decay faster.

The relevant quantities to examine when assessing the possibility of observing wave-packet revivals are 
TABLE II. Parameters of GaAs/ $\mathrm{Al}_{x} \mathrm{Ga}_{1-x}$ As heterostructures, according to Ref. 48.

\begin{tabular}{lcc}
\hline \hline Mass density & $\rho$ & $5300 \mathrm{~kg} / \mathrm{m}^{3}$ \\
Longitudinal sound velocity & $c^{l}$ & $5140 \mathrm{~m} / \mathrm{s}$ \\
Transversal sound velocity & $c^{t}$ & $3040 \mathrm{~m} / \mathrm{s}$ \\
Deformation potential & $D$ & $9.3 \mathrm{eV}$ \\
Piezoelectric constant & $h_{14}$ & $1.2 \times 10^{9} \mathrm{~V} / \mathrm{m}$ \\
\hline \hline
\end{tabular}

$$
\begin{gathered}
\frac{\Gamma_{\tilde{M}}^{-1}}{T_{\mathrm{cl}}} \sim\left(\frac{\tilde{c}}{h_{14}}\right)^{2} \frac{\rho}{\epsilon} \frac{\ln [R / \tilde{M}]}{\widetilde{M}} \sim 100 \frac{\ln [R / \tilde{M}]}{\widetilde{M}}, \\
\frac{\Gamma_{\tilde{M}}^{-1}}{t_{\mathrm{rev}}} \sim\left(\frac{\widetilde{c}}{h_{14}}\right)^{2} \frac{\rho}{\epsilon} \frac{1}{\widetilde{M}^{2}} \sim\left(\frac{7}{\widetilde{M}}\right)^{2} .
\end{gathered}
$$

Equations (39) give, respectively, the number of classical periods and the number of revivals which occur in the mean free time of an EMP WP. We see that, unless $\widetilde{M}$ is very large, the initial periodicity should be observable. However, the likelihood of seeing revivals appears to be quite remote. Equation (39a) shows that the wave packet loses coherence before it can revive. The typical values of parameters for $\mathrm{Al}_{x} \mathrm{Ga}_{1-x} \mathrm{As} / \mathrm{GaAs}$ heterostructures used in the calculation of the EMP WP lifetime are taken from Ref. 48 and are shown in Table II.

\section{Interpretation of previous experiment}

It is interesting to reexamine the experiments described in Ref. 21 in the light of these expressions. In that work measurements were made on a $\mathrm{QH}$ sample in GaAs with the geometry sketched in Fig. 1 at temperature $T=0.3 \mathrm{~K}$. The values of the relevant parameters were filling factor $\nu=1$, magnetic-field strength $B=5.1 \mathrm{~T}$ (i.e., magnetic length $\ell \approx 11 \mathrm{~nm}), R=270 \mu \mathrm{m} \approx 2.4 \times 10^{4} \ell$, and $\zeta_{G} \sim 10 \mu \mathrm{m}$. A single voltage pulse with amplitude $u_{0}=50 \mathrm{mV}$ and duration $T_{\text {exc }}=100$ ps was applied to create the initial wave packet. The latter was observed to move around the disk sample with period $T_{\mathrm{cl}} \approx 4 \mathrm{~ns}$ while spreading rapidly. (Fewer than ten cycles can be discerned before the signal vanishes in the noise.) The data do not seem to consist of a rapid oscillation that is modulated by an envelope.

We believe that the EMP WP excited in this experiment was composed primarily of modes with $M<5$, with $M=1$ possibly having the largest amplitude. Our analysis of the time scales would give $T_{\mathrm{ph}} \approx T_{\mathrm{cl}} \approx t_{\text {rev }} / 20$. In our interpretation of this experiment, the period of the rapid oscillation in the charge signal and the classical period (which determines the periodicity of the envelope function that modulates the rapidly oscillating charge signal) are nearly equal, and the revival time is just one order of magnitude larger. The absence of a rapid oscillation in the charge signal results from the near equality of $T_{\mathrm{ph}}$ and $T_{\mathrm{cl}}$. The decay of the charge signal in $\sim 10$ classical periods can be consistently explained as being due to the spreading of the wave packet due to the nonlinear dispersion of EMP's. However, because of the small value of $\widetilde{M}$ in this experiment, interference between first- and higher-order terms in the expansion of the nonlinear dispersion of the EMP around $\widetilde{M}$ would be expected to (and apparently does) obscure the fractional and full revivals. Exciting the EMP WP with a single voltage pulse results in a rather broad distribution of wave numbers in the EMP WP, so that the analysis of the revival structure, which is based on a sharply peaked distribution of wave numbers around $\widetilde{M}$, is certainly invalid.

With the drift velocity deduced from $R$ and $T_{\mathrm{cl}}$, we estimate the thermal length to be $\zeta_{T} \approx 3 \mu \mathrm{m}$ which is smaller than $\zeta_{G}$. The experiment therefore was in a regime where thermal noise dominates. For a good signal-to-noise ratio, the requirement $u_{0} \gg 2 \mathrm{mV}$ had to be satisfied; this criterion was met in the experiment under consideration. The lifetime of the wave packet as deduced from a calculation outlined in this section is $\sim 1000 T_{\mathrm{cl}}$. Therefore, the rapid decay of the signal cannot be attributed to dissipation into the phonon system.

\section{Nonpolar semiconductors: $\mathrm{Si}$ and $\mathrm{Ge}$}

In nonpolar semiconductors, piezoelectric coupling is absent, and the rate of phonon emission by the plasmons is suppressed for the long-wavelength plasmons typically involved. In this case we find that the ratio of the lifetime and revival time,

$$
\frac{\Gamma_{\tilde{M}}^{-1}}{t_{\mathrm{rev}}} \sim\left[\begin{array}{ll}
\frac{R}{\ln R} & \frac{1}{\widetilde{M}^{2}}
\end{array}\right]^{2},
$$

can be made much larger than unity by adjusting the size of the $\mathrm{QH}$ sample. For a millimeter-size sample at typical magnetic fields $(2 \pi R \approx 1 \mathrm{~mm}, B=10 \mathrm{~T})$, the ratio $\Gamma_{\tilde{M}}^{-1} / t_{\mathrm{rev}} \sim 1$ (25) for $\widetilde{M}=50$ (20). The revival structure of EMP WP disussed in Sec. III should therefore be observable for samples with nonpolar semiconductor host materials, e.g., in $\mathrm{Si} / \mathrm{Ge}$ heterostructures. ${ }^{49}$

\section{SUMMARY AND CONCLUSIONS}

In this paper, we have examined the formation and evolution of edge-magnetoplasmon wave packets in nanostructures. These wave packets are formed as superpositions of edge magnetoplasmons that are the only low-lying excitations in finite quantum-Hall samples. By using a sequence of short pulses in the excitation process, it is possible to produce a superposition with mode numbers sharply peaked around a central value $\widetilde{M}$. We have shown that for such wave packets the initial motion is periodic with a period $T_{\mathrm{cl}}$. After several of these cycles, the wave packet collapses and a sequence of fractional and full revivals commences. This revival structure is analogous to that of Rydberg wave packets in atomic systems; its relevant time scale is the revival time $t_{\text {rev }}$.

We find that experiments that use capacitive coupling to the charge-density fluctuation that is associated with the EMP WP both for the creation and detection of the EMP WP are analogous to Rydberg wave-packet experiments that use the phase-sensitive Ramsey method of detection. In both types of measurement, the semiclassical motion as well as the revival structure is seen in the time variation of the envelope function of a rapidly oscillating signal.

We have shown that thermal effects have no influence on 
the propagation of the wave packet. Examining possible scenarios for the energy loss of EMP's, we found that plasmonphonon coupling due to piezoelectric effects (in polar semiconductors such as GaAs) causes the wave packet to decay with a lifetime that is typically less than the revival time. However, for 2D ES's fabricated in nonpolar semiconductors such as $\mathrm{Si}$ or Ge, piezoelectric coupling is absent and it is possible to produce wave packets with large values of $\widetilde{M}$ that will evolve for times of order $t_{\text {rev }}$ without appreciable decay. In this way, it should be feasible to detect fractional revivals in experiments. The analysis given in this work of a previous experiment ${ }^{21}$ that examined the classical motion of EMP WP's can serve as a guideline for future experimental studies of the EMP WP revival structure.

\section{ACKNOWLEDGMENTS}

We thank R. C. Ashoori, D. DiVincenzo, S. M. Girvin, S. A. Mikhailov, J. J. Palacios, D. Pfannkuche, and R. Hauss- mann for stimulating discussions. This work was funded in part by NSF Grant Nos. DMR-9416906 and PHY-9503756. U.Z. gratefully acknowledges financial support from Studienstiftung des deutschen Volkes (Bonn, Germany).

\section{APPENDIX A: POSSIBLE EXCITATION SCENARIOS FOR THE INITIAL WAVE PACKET}

\section{Single short pulse}

In the experiments on $\mu \mathrm{m}$-size quantum $\operatorname{dots}^{21}$ by Ashoori et al. a single short pulse was applied to prepare the initial wave packet. In our model, the corresponding pulse characteristics is

$$
u(t)= \begin{cases}u_{0} & \text { for } 0 \leqslant t \leqslant T_{\mathrm{exc}} \\ 0 & \text { otherwise }\end{cases}
$$

It is straightforward to calculate the field $B_{ \pm M}(t)$ for this case. We find

$$
B_{ \pm M}(t)=\left\{\begin{array}{l}
0, \quad t \leqslant 0, \\
(\nu M)^{1 / 2} V_{ \pm M}^{\mathrm{ext}} \frac{\mp 2 i u_{0}}{\varepsilon_{M}^{C}} \exp \left[ \pm i \frac{\varepsilon_{M}^{C} t}{2}\right] \sin \left[\frac{\varepsilon_{M}^{C} t}{2}\right], \quad 0<t<T_{\mathrm{exc}}, \\
(\nu M)^{1 / 2} V_{ \pm M}^{\mathrm{ext}} \frac{\mp 2 i u_{0}}{\varepsilon_{M}^{C}} \exp \left[ \pm i \frac{\varepsilon_{M}^{C} T_{\mathrm{exc}}}{2}\right] \sin \left[\frac{\varepsilon_{M}^{C} T_{\mathrm{exc}}}{2}\right], \quad T_{\mathrm{exc}} \leqslant t .
\end{array}\right.
$$

This type of excitation cannot lead to an EMP WP state with a sharply peaked mode distribution and is unlikely to produce well-resolved revivals.

\section{Multiple short pulses}

In analogy with the excitation of Rydberg wave-packet states in atoms by laser pulses, we propose exciting the edge of a $\mathrm{QH}$ system using a series of $N$ short pulses each of duration $T_{\mathrm{exc}}$. For the specific case of sinusoidal individual pulses this would give

$$
u(t)=\left\{\begin{array}{l}
u_{0} \sin \left[\frac{2 \pi}{T_{\mathrm{exc}}} t\right] \text { for } 0 \leqslant t \leqslant N T_{\mathrm{exc}}, \\
0 \text { otherwise }
\end{array}\right.
$$

In this case we find that for $t \geqslant N T_{\text {exc }}$

$$
\begin{aligned}
B_{ \pm M}(t)= & (\nu M)^{1 / 2} V_{ \pm M}^{\mathrm{ext}} \frac{4 \pi u_{0}}{T_{\mathrm{exc}}} \\
& \times \exp \left[ \pm i \frac{\varepsilon_{M}^{C} N T_{\mathrm{exc}}}{2}\right] \frac{\sin \left[\varepsilon_{M}^{C} N T_{\mathrm{exc}} / 2\right]}{\left(\varepsilon_{M}^{C}\right)^{2}-\left(\frac{2 \pi}{T_{\mathrm{exc}}}\right)^{2}},
\end{aligned}
$$

which is sharply peaked around a value $\widetilde{M}$ satisfying $\varepsilon_{\widetilde{M}}^{C}=2 \pi / T_{\text {exc }}$. Using our notation from Sec. III B, the EMP WP's created with the multiple-short-pulse technique satisfy
$T_{\text {ph }} \equiv T_{\text {exc }}$. The duration of the short pulses determines the value of $\widetilde{M}$, whereas the number of pulses $N$ determines the width of the peak in $B_{ \pm M}(t)$ at $\tilde{M}$. Figure 2 illustrates some possible distributions in $M$ that could be produced using this method.

\section{Adiabatic limit}

An important limit of our general results is the case of an adiabatically varying potential $V^{\text {ext }}(t)$. In our formalism, this corresponds to a pulse characteristics $u(t)$ which varies on a time scale longer than the time scale set by the lowest EMP energy. The time integral in expression Eq. (13) is then dominated by the exponential, and $u(\tau)$ can be treated as a constant within the range of integration. We find

$$
B_{ \pm M}(t)=(\nu M)^{1 / 2} V_{ \pm M}^{\mathrm{ext}} \frac{-u(t)}{\varepsilon_{M}^{C}} \exp \left[ \pm i \varepsilon_{M}^{C} t\right]
$$

and the total density response $\widetilde{\varrho}_{M}(t)$, derived from Eq. $(14 \mathrm{c})$, is

$$
\tilde{\varrho}_{M}(t)=-u(t) V_{M}^{\mathrm{ext}} \frac{\nu M}{\varepsilon_{M}^{C}} .
$$

The induced density in Eq. (A6) is the instantaneous ground-state density that minimizes the energy in the presence of the slowly varying external potential. The energy in 
the presence of the external potential can be expressed in terms of the charge density as follows:

$$
\begin{aligned}
E[\varrho]= & \int d \theta \int d \theta^{\prime} V^{\mathrm{int}}\left(\theta, \theta^{\prime}\right) \widetilde{\varrho}(\theta) \widetilde{\varrho}\left(\theta^{\prime}\right) \\
& +u(t) \int d \theta V^{\operatorname{ext}}(\theta) \widetilde{\varrho}(\theta) .
\end{aligned}
$$

The configuration that minimizes the energy functional is

$$
\widetilde{\varrho}_{M}(t)=-u(t) \frac{V_{M}^{\mathrm{ext}}}{V_{M}^{\mathrm{int}}}=-u(t) V_{M}^{\mathrm{ext}} \frac{\nu M}{\varepsilon_{M}^{C}},
$$

consistent with Eq. (A6).

\section{APPENDIX B: DERIVATION OF THE EFFECTIVE EMP-PHONON COUPLING}

In this section, the derivation of the effective coupling between the EMP's and 3D phonons is given. We start with the full Hamiltonian [Eq. (34)] describing the interaction between 3D electrons with the 3D lattice in the sample. It is convenient to study the Fourier components of the 3D electron density

$$
\varrho_{\vec{q}}^{3 \mathrm{D}}=\int d^{3} r e^{i \vec{q} \cdot \vec{r}} \varrho^{3 \mathrm{D}}(\vec{r})
$$

As we are dealing with a 2D ES which is confined, say, to the $x y$ plane, we introduce the notation $\vec{r}=z \hat{z}+r$, where $\hat{z} \perp r$ (in reciprocal space: $\vec{q}=q_{z} \hat{z}+Q, \hat{z} \perp Q$ ) and assume the electron density to be peaked strongly at $z=z_{0}$ : $\varrho^{3 \mathrm{D}}(\vec{r})=\chi(z) \varrho^{2 \mathrm{D}}(r)$. Then, the $z$ integration in Eq. (B1) decouples from the rest of the 3D integral, and merely leads to a form factor $F^{\perp}\left(q_{z}\right)=\int d z e^{i z \cdot q_{z}} \chi(z)$. We are left with the $2 \mathrm{D}$ Fourier transform of the $2 \mathrm{D}$ electron density $\varrho^{2 \mathrm{D}}(r)$.

\section{Disk geometry}

Specializing to the case of a $\mathrm{QH}$ sample in the disk geometry (see Fig. 1), we can write approximately

$$
\varrho^{2 \mathrm{D}}(r)=\varrho_{0} \Theta(R-|r|)+\varrho^{1 \mathrm{D}}(\theta) \delta(R-|r|),
$$

where $\varrho_{0}=\nu / 2 \pi, \Theta(x)$ is the Heaviside step function, and $\theta$ is the coordinate along the edge (see Fig. 1). The remaining integrals can be performed. The result is

$$
\begin{gathered}
\varrho_{\vec{q}}^{3 \mathrm{D}}=\varrho_{\vec{q}}^{\text {bulk }}+\varrho_{\vec{q}}^{\text {edge }} \\
\varrho_{\vec{q}}^{\text {bulk }}=F^{\perp}\left(q_{z}\right) \nu R^{2} J_{1}(Q R), \\
\varrho_{\vec{q}}^{\text {edge }}=F^{\perp}\left(q_{z}\right) \sum_{M>0} i^{M} J_{M}(Q R)[\nu M]^{1 / 2}\left(b_{M}^{\dagger} e^{-i M \theta_{q}^{\vec{q}}}\right. \\
+b_{M} e^{\left.i M \theta_{q}^{\vec{q}}\right)}
\end{gathered}
$$

where we write $\theta_{\vec{q}}$ for the polar angle of the vector $Q$ in the $x y$ plane, i.e., the azimuthal angle of $\vec{q}$ in 3D. We remind the reader that $Q$ is the projection of the wave vector $\vec{q}$ onto the plane where the 2D ES is located. To get Eq. (B3c), we inserted Eq. (2) for $\varrho^{1 \mathrm{D}}(\theta)$. Using expression Eq. (B3a) for the 3D electron density $\varrho_{q}^{3 \mathrm{D}}$ in Eq. (34), we find the Hamiltonian Eq. (35) for the coupling between the EMP's and phonon modes, with

$$
C_{M}^{\lambda}(\vec{q})=[\nu M]^{1 / 2} M^{\lambda}(\vec{q}) i^{M} J_{M}(Q R) \quad F^{\perp}\left(q_{z}\right)
$$

as the coupling strength. Note that $J_{M}(x)$ denotes the $M$ th-order Bessel function of the first kind.

\section{Strip geometry}

For the sake of completeness, we give the corresponding results for the case of a QH bar (strip geometry). By QH bar, we mean a sample with periodic boundary conditions applied in the $\hat{x}$ direction, and open boundary conditions in the $\hat{y}$ direction. This configuration space corresponds to the surface of a cylinder with the axis in the $\hat{y}$ direction. Although this geometry is not appropriate for the observation of EMP WP revivals, it can be useful in analyzing experiments in which edge disturbances travel along the edge of a Hall bar. ${ }^{22}$

In analogy with Eq. (B2), we write for the case of the strip geometry

$$
\varrho^{2 \mathrm{D}}(r)=\varrho_{0} \Theta(W-y)+\varrho^{1 \mathrm{D}}(\theta) \quad \delta(y-W),
$$

with $W$ denoting the width of the strip. We again end up with an expression like Eq. (B3a), and find for the Hamiltonian describing the plasmon-phonon coupling:

$$
\begin{aligned}
H^{\mathrm{pl}-\mathrm{ph}}= & \sum_{\substack{M>0 \\
\vec{q}, \lambda}} C(\vec{q}, \lambda)\left(a_{\vec{q}, \lambda}+a_{-\vec{q}, \lambda}^{\dagger}\right)\left(b_{M} \delta_{M,-R \cdot q_{X}}\right. \\
& \left.+b_{M}^{\dagger} \delta_{M, R \cdot q_{x}}\right) .
\end{aligned}
$$

The coupling strength is

$$
C(\vec{q}, \lambda)=[\nu M]^{1 / 2} M^{\lambda}(\vec{q}) \quad F^{\perp}\left(q_{z}\right) e^{i W \cdot q_{y}},
$$

which is different from the result Eq. (B4) that we found for the disk case. Note that the factor $e^{i W \cdot q_{y}}$ is simply a form factor describing the profile of the charge density in the $\hat{y}$ direction. Here, we have assumed a sharp confining potential and therefore used a $\delta$-function. In general, this is not an experimentally realistic situation, and we will have to replace $e^{i W \cdot q_{y}}$ by a form factor $F^{\|}\left(q_{y}\right)$. A similar form factor should in principle be included in the analysis for the disk geometry as well, but would not be important for small $M$. Note the differences between the final expression for the coupling of EMP's to phonons, Eq. (35) for the disk geometry, and Eq. (B6) the for strip geometry.

\section{Specialization: Acoustic phonons in semiconductors}

For the physical situation we are concerned with in this paper, acoustic phonons play the dominant role. In polar semiconductors, as for instance in $\mathrm{Al}_{x} \mathrm{Ga}_{1-x} \mathrm{As} / \mathrm{GaAs}$ heterostructures, phonon coupling occurs due to both deformationpotential and piezoelectric scattering. The bare 3D electronphonon coupling reads 


$$
M^{\lambda}(\vec{q})=\left(\frac{\hbar}{2 \rho \Omega[\epsilon(Q)]^{2} \omega^{\lambda} \vec{q}}\right)^{1 / 2}\left[D q \delta_{\lambda, l}+i e h_{14} \mathcal{M}_{\lambda}(\hat{q})\right],
$$

where $\rho$ and $\Omega$ denote the 3D bulk density and the sample volume, respectively. We have also introduced the strengths of the deformation potential $(D)$ and the piezoelectric coupling $\left(h_{14}\right)$. For more details on phonons in $\mathrm{Al}_{x} \mathrm{Ga}_{1-} \mathrm{As} / \mathrm{GaAs}$ heterostructures as well as numerical values of the parameters, see Ref. 48. A general reference on scattering mechanisms in metals and semiconductors is Ref. 50. The dielectric function $\epsilon(Q)$ incorporates screening of the original electron-phonon interaction due to manyelectron effects. Here, the 2D ES is in the QH regime, and there is no screening, therefore we set $\epsilon(Q) \rightarrow 1$. Finally, the functions $\mathcal{M}_{\lambda}(\hat{q})$ model the directional dependence of the piezoelectric phonon coupling. For a 2D ES which lies in the (100) plane of GaAs, we have ${ }^{48}$

$$
\begin{gathered}
{\left[\mathcal{M}_{l}(\hat{q})\right]^{2}=\frac{9}{2} \frac{Q^{4} q_{z}^{2}}{q^{6}},} \\
{\left[\mathcal{M}_{t}(\hat{q})\right]^{2}=2 \frac{Q^{2} q_{z}^{4}}{q^{6}}+\frac{1}{4} \frac{Q^{6}}{q^{6}},}
\end{gathered}
$$

for longitudinal and transverse modes, respectively.

\section{APPENDIX C: PLASMON SELF-ENERGY DUE TO PHONON COUPLING}

Due to the plasmon-phonon coupling, the plasmons acquire a nonvanishing imaginary part of the self-energy that is related to the rate of phonon emission/absorption by the plasmons. Here, we calculate the plasmon self-energy using a diagrammatic perturbation theory. Due to azimuthal symmetry, the self-energy is diagonal in angular momentum indices. As the Hamiltonian of the coupled EMP-phonon system is quadratic, the leading-order diagram gives the exact result for the self-energy. Note that here we consider the coupling of chiral 1D plasmons to 3D phonons; the problem of chiral 1D plasmons coupled to 1D phonons with implications for quantum-Hall edges has been discussed previously. ${ }^{51}$

The full Hamiltonian of the coupled EMP-phonon system (without the external potential forming the initial wave packet) is

$$
H^{\prime}=H_{0}^{\mathrm{pl}}+H_{0}^{\mathrm{ph}}+H^{\mathrm{pl}-\mathrm{ph}},
$$

where $H_{0}^{\mathrm{pl}}$ is given by Eq. (8), the expression Eq. (35) for $H^{\mathrm{pl}-\mathrm{ph}}$ in the disk geometry has been derived in Appendix B, and $H_{0}^{\mathrm{ph}}$ describes a system of free 3D phonons with dispersion relation $\omega_{q}^{\lambda}$ :

$$
H_{0}^{\mathrm{ph}}=\sum_{\vec{q}, \lambda} \omega_{\vec{q}}^{\lambda} a_{\vec{q}, \lambda}^{\dagger} a_{\vec{q}, \lambda} .
$$

We want to calculate the single-plasmon Matsubara Green's function defined in Eq. (36), which can be written as a sum (a)

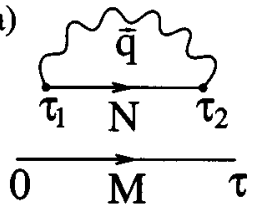

(b)

(c)

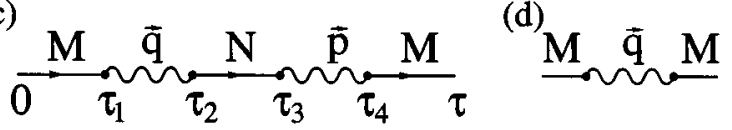

FIG. 7. Diagrams involved in the calculation of the plasmon propagator (a, b, c) and the plasmon self-energy (d), arising from plasmon-phonon coupling. Straight lines denote the free plasmon propagator $\mathcal{G}_{M}^{0}(\tau)$. The wavy line is the usual phonon propagator $\mathcal{D}_{\lambda}^{0}\left(\vec{q}, \tau_{1}-\tau_{2}\right)$. (a) Disconnected diagram; does not contribute to $\mathcal{G}_{M}(\tau)$. (b) Connected diagram; leading contribution to $\mathcal{G}_{M}(\tau)$. (c) Higher-order contribution to the plasmon propagator. Due to azimuthal symmetry such a diagram vanishes unless $M=N$. The selfenergy insertion in this diagram is improper. The proper self-energy is diagonal in angular-momentum indices and consists of the single diagram shown in $(d)$.

over all distinct connected diagrams. Three of the diagrams appearing in $\mathcal{G}_{M}(\tau)$ are shown in Fig. 7. All diagrams of higher than second order are reducible. The sums over phonon wave vectors that are implicit in the diagrams enforce the conservation of the plasmon wave number in each higher-order diagram. Using the standard definition ${ }^{47}$ for the phonon propagator

$$
\begin{aligned}
\mathcal{D}_{\lambda}^{0}\left(\vec{q}, \tau_{1}-\tau_{2}\right)= & -\left\langleT _ { \tau } [ a _ { - \vec { q } , \lambda } ^ { \dagger } ( \tau _ { 1 } ) + a _ { \vec { q } , \lambda } ( \tau _ { 1 } ) ] \left[ a_{\vec{q}, \lambda}^{\dagger}\left(\tau_{2}\right)\right.\right. \\
& \left.\left.+a_{-\vec{q}, \lambda}\left(\tau_{2}\right)\right]\right\rangle_{0},
\end{aligned}
$$

we find that the full plasmon propagator is given exactly by

$$
\mathcal{G}_{M}\left(i \omega_{n}\right)=\left[i \omega_{n}-\varepsilon_{M}^{C}-\Sigma_{M}\left(i \omega_{n}\right)\right]^{-1},
$$

with the plasmon self-energy

$$
\Sigma_{M}\left(i \omega_{n}\right)=\sum_{\vec{q}, \lambda}\left|C_{M}^{\lambda}(\vec{q})\right|^{2} \mathcal{D}_{\lambda}^{0}\left(\vec{q}, i \omega_{n}\right),
$$

which is the contribution from second-order perturbation theory, expressed diagrammatically in Fig. 7(d). The same result for the plasmon propagator is obtained when integrating out the phonon degrees of freedom in a path-integral expression for the partition function of the coupled EMPphonon system.

After continuation to real frequencies, it is possible to find the imaginary part of the self-energy for the retarded Green's function

$$
\operatorname{Im} \Sigma_{M}(\omega)=\pi \sum_{\vec{q}, \lambda}\left|C_{M}^{\lambda}(\vec{q})\right|^{2}\left[\delta\left(\omega+\omega_{\vec{q}}\right)-\delta\left(\omega-\omega_{\vec{q}}\right)\right] .
$$

From this expression, we read off the damping rate for the mode with wave number $M$ as expressed in Eq. (37). 
${ }^{1}$ A. H. MacDonald, in Proceedings of the 1994 Les Houches Summer School on Mesoscopic Quantum Physics, edited by E. Akkermans et al. (Elsevier Science, Amsterdam, 1995), pp. 659720.

${ }^{2}$ A. H. MacDonald, Phys. Rev. Lett. 64, 220 (1990).

${ }^{3}$ A. H. MacDonald, Brazilian J. Phys. 26, 43 (1996).

${ }^{4}$ X. G. Wen, Phys. Rev. B 41, 12838 (1990).

${ }^{5}$ X. G. Wen, Phys. Rev. B 44, 5708 (1991).

${ }^{6}$ X. G. Wen, Int. J. Mod. Phys. B 6, 1711 (1992).

${ }^{7}$ V. J. Emery, in Highly Conducting One-Dimensional Solids, edited by J. T. Devreese et al. (Plenum Press, New York, 1979), pp. 247-303.

${ }^{8}$ J. Sólyom, Adv. Phys. 28, 201 (1979).

${ }^{9}$ F. D. M. Haldane, J. Phys. C 14, 2585 (1981).

${ }^{10}$ D. B. Mast, A. J. Dahm, and A. L. Fetter, Phys. Rev. Lett 54, 1706 (1985).

${ }^{11}$ Edge-magnetoplasmon collective excitations of $2 \mathrm{D}$ electron systems occur quite generally but, outside of the quantum-Hall regime, they have a rather short lifetime, and the excitation spectrum does not completely bosonize. In macroscopic systems they are generally strongly overdamped in strong magnetic fields, except near the filling factors at which the quantum-Hall effect occurs. For early theoretical studies, see A. L. Fetter, Phys. Rev. B 32, 7676 (1985); J.-W. Wu, P. Hawrylak, and J. J. Quinn, Phys. Rev. Lett. 55, 879 (1985); and Ref. 12. For a review and additional references, see V. A. Volkov and S. A. Mikhailov, in Landau Level Spectroscopy, edited by G. Landwehr and E. I. Rashba (Elsevier, Amsterdam, 1991), pp. 855907.

${ }^{12}$ V. A. Volkov and S. A. Mikhailov, Pis'ma Zh. Éksp. Teor. Fiz. 42, 450 (1985) [JETP Lett. 42, 556 (1985)]; Zh. Éksp. Teor. Fiz. 94, 217 (1988) [Sov. Phys. JETP 67, 1639 (1988)].

${ }^{13}$ U. Zülicke and A. H. MacDonald, Phys. Rev. B 54, 16813 (1996)

${ }^{14}$ M. Wassermeier et al., Phys. Rev. B 41, 10287 (1990).

${ }^{15}$ Ya. M. Blanter and Yu. E. Lozovik, Physica B 182, 254 (1992).

${ }^{16}$ The integer $M$ labels the fundamental modes at the edge of the $\mathrm{QH}$ sample (i.e., the EMP modes). For a disk-shaped QH sample, $M$ has the meaning of the angular momentum quantum number. If we think of the $\mathrm{QH}$ sample as a semi-infinite cylinder (i.e., periodic boundary conditions are applied to the edge), $M$ can be identified with the (linear) momentum along the edge.

${ }^{17}$ S. J. Allen, H. L. Störmer, and J. C. M. Hwang, Phys. Rev. B 28, 4875 (1983).

${ }^{18}$ V. I. Tal'yanskiǔ et al., Pis'ma Zh. Éksp. Teor. Fiz. 50, 196 (1989) [JETP Lett. 50, 221 (1989)].

${ }^{19}$ T. Demel, D. Heitmann, P. Grambow, and K. Ploog, Phys. Rev. Lett. 64, 788 (1990).

${ }^{20}$ I. Grodnensky, D. Heitmann, and K. von Klitzing, Phys. Rev. Lett. 67, 1019 (1991).

${ }^{21}$ R. C. Ashoori et al., Phys. Rev. B 45, 3894 (1992).

${ }^{22}$ N. B. Zhitenev, R. J. Haug, K. v. Klitzing, and K. Eberl, Phys. Rev. Lett. 71, 2292 (1993); G. Ernst, R. J. Haug, J. Kuhl, K. v. Klitzing, and K. Eberl, ibid. 77, 4245 (1996).

${ }^{23}$ D. C. Glattli et al., Phys. Rev. Lett 54, 1710 (1985).

${ }^{24}$ P. J. M. Peters et al., Phys. Rev. Lett. 67, 2199 (1991).

${ }^{25}$ J. Parker and C. R. Stroud, Phys. Rev. Lett. 56, 716 (1986).

${ }^{26}$ G. Alber, H. Ritsch, and P. Zoller, Phys. Rev. A 34, 1058 (1986); G. Alber and P. Zoller, Phys. Rep. 199, 231 (1991).

${ }^{27}$ A. ten Wolde, L. D. Noordam, A. Lagendijk, and H. B. van Linden van den Heuvell, Phys. Rev. Lett. 61, 2099 (1988).
${ }^{28}$ J. A. Yeazell, M. Mallalieu, J. Parker, and C. R. Stroud, Phys. Rev. A 40, 5040 (1989); J. A. Yeazell, M. Mallalieu, and C. R. Stroud, Phys. Rev. Lett. 64, 2007 (1990); J. A. Yeazell and C. R. Stroud, Phys. Rev. A 43, 5153 (1991).

${ }^{29}$ D. R. Meacher, P. E. Meyler, I. G. Hughes, and P. Ewart, J. Phys. B 24, L63 (1991).

${ }^{30}$ I. Sh. Averbukh and N. F. Perelman, Phys. Lett. A 139, 449 (1989); C. Leichtle, I. Sh. Averbukh, and W. P. Schleich, Phys. Rev. Lett. 77, 3999 (1996).

${ }^{31}$ M. Nauenberg, J. Phys. B 23, L385 (1990).

${ }^{32}$ R. Bluhm and V. A. Kostelecký, Phys. Rev. A 50, R4445 (1994); Phys. Lett. A 200, 308 (1995); Phys. Rev. A 51, 4767 (1995).

${ }^{33}$ See, for example, R. Bluhm, V. A. Kostelecký, and J. Porter, Am. J. Phys. 64, 944 (1996).

${ }^{34}$ R. Bluhm, V. A. Kostelecký, and B. Tudose, Phys. Lett. A 222, 220 (1996); Phys. Rev. A 55, 819 (1997).

${ }^{35}$ R. Bluhm and V. A. Kostelecký, Phys. Rev. A 48, R4047 (1993); 49, 4628 (1994); R. Bluhm, V. A. Kostelecký, and B. Tudose, ibid. 52, 2234 (1995); 53, 937 (1996).

${ }^{36}$ V. A. Kostelecký and M. M. Nieto, Phys. Rev. Lett. 53, 2285 (1984); Phys. Rev. A 32, 1293 (1985); 32, 3243 (1985); R. Bluhm and V. A. Kostelecký, ibid. 47, 794 (1993).

${ }^{37}$ J. Wals, H. H. Fielding, J. F. Christian, L. C. Snoek, W. J. van der Zande, and H. B. van Linden van den Heuvell, Phys. Rev. Lett. 72, 3783 (1994).

${ }^{38}$ L. D. Noordam, D. I. Duncan, and T. F. Gallagher, Phys. Rev. A 45, 4734 (1992).

${ }^{39}$ B. Broers, J. F. Christian, J. H. Hoogenraad, W. J. van der Zande, H. B. van Linden van den Heuvell, and L. D. Noordam, Phys. Rev. Lett. 71, 344 (1993).

${ }^{40}$ J. F. Christian, B. Broers, J. H. Hoogenraad, W. J. van der Zande, and L. D. Noordam, Opt. Commun. 103, 79 (1993).

${ }^{41}$ N. F. Ramsey, Molecular Beams (Oxford University Press, Oxford, 1956).

${ }^{42}$ There exists an analogy between the charge signal $Q(t)$ that is induced by the charge density of a moving EMP WP at a detecting gate and the observable that is employed to measure Rydberg wave packets using Ramsey's method (i.e., the ionization signal). Both signals consist of a rapidly oscillating part which is modulated by an envelope function, and it is the latter that shows a classical behavior on short times and a revival structure on longer times.

${ }^{43}$ Note that the existence of the rapid oscillations necessarily follows from the observability criterion for revivals: $t_{\text {rev }} \gg T_{\mathrm{cl}}$ implies $T_{\mathrm{cl}} \gg T_{\mathrm{ph}}$ (which is equivalent to $\widetilde{M} \gg 1$ ). The chargedensity configuration of the EMP WP consists of a finite classical wave train which has a large dominant wave number $\widetilde{M}$. In this it differs from a Rydberg wave packet which is a complex wave.

${ }^{44}$ See, for example, J. D. Jackson, Classical Electrodynamics, Second ed. (Wiley, New York, 1975), p. 582.

${ }^{45}$ Strictly speaking, the classical period $T_{\mathrm{cl}}$ is not entirely determined by classical physics. This is a difference between the physics of EMP WP's and Rydberg wave packets. As the EMP WP state is a many-body quantum state which is a superposition of many-electron collective modes (EMP's), its time evolution is influenced by exchange-correlation effects among the electrons in the 2D ES. For the case of long-range interaction, these effects become more important at larger wave numbers $M$. However, for macroscopic QH samples, because of long-range interactions the influence of exchange-correlation effects on the EMP 
dispersion is relatively small (Ref. 13), and we recover approximately the classical drift period.

${ }^{46}$ D. C. Tsui, H. L. Störmer, and A. C. Gossard, Phys. Rev. B 25, 1405 (1982).

${ }^{47}$ G. D. Mahan, Many-Particle Physics (Plenum, New York, 1990).

${ }^{48}$ S. K. Lyo, Phys. Rev. B 38, 6345 (1988), and references therein.

${ }^{49}$ For measurements of the quantum-Hall effect in $\mathrm{Si} / \mathrm{Ge}-$ heterostructures, see, e.g., D. Többen, F. Schäffer, A. Zrenner, and G. Abstreiter, Phys. Rev. B 46, 4344 (1992); R. B. Dunford et al., Surf. Sci. 361/362, 550 (1996); K. Ismail, Physica B 227, 310 (1996). A brief review of $\mathrm{Si} / \mathrm{Ge}$ heterostructures, including more references, can be found in G. Abstreiter, Solid State Commun. 92, 5 (1994).

${ }^{50}$ V. F. Gantmakher and Y. B. Levinson, Carrier Scattering in Metals and Semiconductors (North Holland, Amsterdam, 1987).

${ }^{51}$ O. Heinonen and S. Eggert, Phys. Rev. Lett. 77, 358 (1996). 